CERN-TH/96-186

hep-th/9607146

\title{
Relic Gravitons from the Pre-Big Bang: What we Know and What we Do Not Know *
}

\author{
M. Gasperini \\ Theory Division, CERN, CH-1211 Geneva 23, Switzerland \\ and \\ Dipartimento di Fisica Teorica, Università di Torino, \\ Via P. Giuria 1, 10125 Turin, Italy
}

\begin{abstract}
I discuss the status of present knowledge about a possible background of relic gravitons left by an early, pre-big bang cosmological epoch, whose existence in the past of our Universe is suggested by the duality symmetries of string theory.
\end{abstract}

To appear in

"New developments in string gravity and physics at the Planck energy scale" ed. by N. Sanchez (World Scientific, Singapore, 1996)

CERN-TH/96-186

July 1996

*Based on talks presented at the Meeting on "Detection of high-frequency gravitational waves" (CERN, January 1996), at the INTAS Meeting on "Fundamental problems in classical, quantum and string gravity" (Turin, May 1996), at the "XLth Conference of the Italian Astronomical Society" (Osservatorio di Roma, Rome, May 1996), and at the Network Meeting on "String Gravity" (Observatoire de Paris, June 1996). 



\title{
RELIC GRAVITONS FROM THE PRE-BIG BANG: WHAT WE KNOW AND WHAT WE DO NOT KNOW
}

\author{
MAURIZIO GASPERINI \\ Theory Division, CERN, CH-1211 Geneva 23, Switzerland \\ and Dipartimento di Fisica Teorica, Università di Torino, Turin, Italy
}

I discuss the status of present knowledge about a possible background of relic gravitons left by an early, pre-big bang cosmological epoch, whose existence in the past of our Universe is suggested by the duality symmetries of string theory.

\section{Table of Contents}

1. Introduction

2. The Pre-Big Bang Scenario

2.1. Motivations

2.2. Kinematical aspects

3. Growth of Perturbations

3.1. Formal problems

3.2. Phenomenological consequences

4. The Expected Graviton Background

4.1. Minimal model

4.2. A diagrammatic approach to the perturbation spectrum

4.3. Constraints from photon production

4.4. Non-minimal models

4.5. Experimental sensitivities

5. Conclusion

Appendix A: The dimensionless amplitude $\left|\delta_{h}\right|$

Appendix B: The spectral amplitude $S_{h}$ 


\section{Introduction}

The cosmological solutions of the low energy string effective action, and their symmetry properties, have recently motivated the study of a very early, "pre-big bang" cosmological phase睓, whose kinematical properties are the "dual" counterpart of the present, standard cosmological phase. The purpose of this paper is to give a short introduction to the pre-big bang scenario, to explain why the amplification of perturbations is more efficient in such a context than in the standard inflationary scenario, and to discuss the general properties of a possible cosmic graviton background associated to the transition from the pre- to the post-big bang epoch.

The main ideas and results reported here are the fruit of a research programme in string cosmology that started from earlier work done with Norma Sanchez and Gabriele Veneziano on the motion of strings in accelerated backgrounds 45 , and that was subsequently developed in collaboration with Gabriele Veneziano and with a number of other researchers: Ramy Brustein, Massimo Giovannini, Jnan Maharana, Krzysz Meissner and Slava Mukhanov (listed here in alphabetical order). The interested reader can find an updated list of references and papers about the pre-big bang scenario on the WWW "home page" devoted to string cosmology, available at the address:

http://www.to.infn.it/teorici/gasperini/

\section{The Pre-Big Bang Scenario}

I will call, for short, pre-big bang scenario, a cosmological scenario that is based on the equations obtained from the string effective action and which includes an initial phase of accelerated evolution and growing curvature 1 . B. The most revolutionary aspect of the class of models describing this scenario is probably the fact that the initial state of the Universe, instead of being hot and dense as in the standard cosmological context, is the string perturbative vacuum, namely a state with flat metric, vanishing gauge coupling $\left(g=e^{\phi / 2}=0, \phi=-\infty\right)$, and no matter present (with the possible exception of an incoherent, highly diluted gas of non-interacting strings 3 ). As a consequence, in this class of models the initial evolution of the Universe can be consistently described in terms of the lowest order string effective action,

$$
\begin{aligned}
S= & -\int d^{d+1} x \sqrt{|g|}\left[e^{-\phi}\left(R+(\nabla \phi)^{2}+\alpha^{\prime} \text { corrections }\right)+V(\phi, \text { nonperturbative })\right] \\
& +\operatorname{loops}\left(e^{\phi}\right),
\end{aligned}
$$

neglecting both finite-size effects ( $\alpha^{\prime}$ corrections) and higher (field theory) loops in the coupling constant $g=e^{\phi / 2}$. The initial evolution is simply driven by the kinetic energy of the dilaton field, with negligible contributions also from a possible non-perturbative potential $V(\phi)$, which is known, at small coupling, to approach 
zero very rapidly 6 as $V \sim \exp [-\exp (-\phi)]$. Here I will neglect, for simplicity, also a possible anisotropic contribution of the string antisymmetric tensor to the cosmological equations; see Ref. [7] for the inclusion of such a contribution.

Irrespective of the possible initial presence of matter sources23, the (generally anisotropic) solution of the string cosmology equations, after inserting the perturbative vacuum as initial condition, can be asymptotically parametrized as

$$
a_{i}=(-t)^{\beta_{i}}, \quad \phi=\left(\sum_{i} \beta_{i}-1\right) \ln (-t), \quad \sum_{i} \beta_{i}^{2}=1, \quad t<0, \quad t \rightarrow 0
$$

$(i=1, . ., d)$. This solution describes a phase in which the curvature scale and the dilaton are both growing, since $\left|H_{i}\right| \sim(-t)^{-1} \sim|\dot{\phi}|$ for $t \rightarrow 0_{-}$, where $H=\dot{a} / a$, and the dot denotes derivatives with respect to cosmic time $t$. A typical example of background is illustrated in Fig. 1.
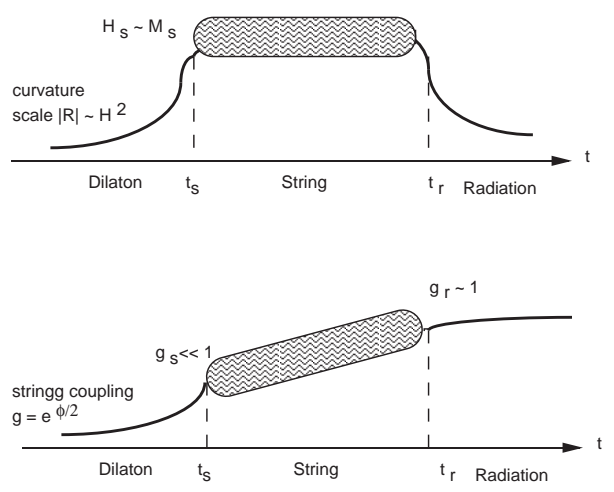

Figure 1: Qualitative evolution of the curvature scale and of the string coupling as a function of time, for a generic pre-big bang type of background.

Of course, when the curvature reaches the string scale, determined by the string mass parameter $M_{s} \equiv \lambda_{s}^{-1}$, the background enters a truly "stringy" regime, in which all higher derivative terms in the $\alpha^{\prime}$ expansion become important (our present imperfect knowledge of the detailed evolution, in that region, is represented by the wavy lines of Fig. 1). At the end of the string phase the dilaton eventually approaches the strong coupling regime, $g_{r} \equiv g\left(t_{r}\right) \sim 1$, where both the quantum loop effects and the semiclassical back-reaction of the produced radiation become important, and the transition to the standard decelerated evolution (with frozen dilaton) is expected to occur. Some concrete examples of how the inclusion of oneloop corrections can stop the growth of the curvature have been presented recently and discussed in the context of four- 2 and two-dimensional 1 cosmology.

At the beginning of the string phase, on the contrary, the value of the string coupling $g_{s} \equiv g\left(t_{s}\right)$ can be arbitrarily small (see Fig. 1); it represents one of the main parameters of this class of backgrounds. The other important parameter is the duration of the string phase, measured by the ratio $\left|t_{s} / t_{r}\right|$, or by the ratio of 
the associated scale factors, $a\left(t_{s}\right) / a\left(t_{r}\right)$. If $g_{s}$ is already of order 1 at the beginning of the string phase, the duration of such a phase presumably shrinks to a number of order unity (in string units). The presence of such a phase, however, cannot be avoided; otherwise, to the tree-level in $g_{s}$, and to zeroth order in $\alpha^{\prime}$, the growth of the curvature and of the dilaton is unbounded 11. Even in that case, however, one can compute quantum-mechanically, via the Wheeler-De Witt equation, the transition probability from the initial to the final background configuration, Such a transition can be represented as a wave reflection in minisuperspace 12,23 , and the probability turns out to be finite and non-vanishing even in the presence of a singularity that disconnects, classically, the two asymptotic regimes.

\subsection{Motivations}

There are at least two motivations, in a string theory context, that lead to introduce an early, pre-big bang cosmological phase in the past history of our Universe, as a natural complement of the present, post-big bang cosmological era. The first motivation is provided by the solutions of the classical string equations of motion embedded in inflationary cosmological backgrounds event horizons, a gas of classical string develops in fact an effective negative pressure, so that the background evolution can be consistently self-sustained by the string gas itself as a source3.14.

The second motivation is provided by the duality symmetries of the lowest-order string effective action, which can be written, including the antisymmetric tensor $B_{\mu \nu}$,

$$
S=-\int d^{d+1} x \sqrt{|g|} e^{-\phi}\left[R+\left(\nabla_{\mu} \phi\right)^{2}-\frac{1}{2}\left(\partial_{[\mu} B_{\nu \alpha]}\right)^{2}\right], \quad \mu, \nu=1,2, \ldots, d+1 .
$$

If $a(t)$ and $\phi(t)$ represent an exact, homogeneous and isotropic solution of this action (with $B=0$ and zero spatial curvature), then

$$
\tilde{a}=a^{-1}, \quad \tilde{\phi}=\phi-2 d \ln a
$$

represent another exact solution, thanks to the scale factor duality symmetry 15,16 . This symmetry is only a particular case of a more general covariance property of the action (2.3) under global $O(d, d)$ transformations 17.18 , which mixes non-trivially the spatial components of the metric and of the antisymmetric tensor,

$$
M \rightarrow \tilde{M}=\Lambda^{T} M \Lambda, \quad M=\left(\begin{array}{cc}
G^{-1} & -G^{-1} B \\
B G^{-1} & G-B G^{-1} B
\end{array}\right)
$$

$\left(\Lambda \in O(d, d)\right.$ and $\left.G=g_{i j}, B=B_{i j}\right)$. Such an $O(d, d)$ covariance is also preserved by the addition of source terms to the cosmological equations 19 , provided they represent "bulk" string matter, whose components satisfy the string equation of motion in the given background. In the presence of sources, and in the perfect fluid approximation, a scale factor duality transformation is simply associated to a "reflection" of the equation of state $15, p / \rho \rightarrow-p / \rho$. 
The importance of these symmetriest, in our cosmological context, follows from the fact that by operating simultaneously a duality transformation (for instance according to eq. (2.4) ) and a time-reversal transformation on a given decelerated, post-big bang solution,

$$
\dot{a}>0, \quad \ddot{a}<0, \quad \dot{H}<0,
$$

it is always possible to obtain a new accelerated solution, with growing curvature, of the pre-big bang type:

$$
\dot{a}>0, \quad \ddot{a}>0, \quad \dot{H}>0 .
$$

In $d=3$, for instance, the standard radiation-dominated solution

$$
a=t^{1 / 2}, \quad \phi=\text { const }, \quad p=\rho / 3, \quad t>0
$$

(which is still an exact solution for the action (2.3) supplemented by perfect fluid sources) is mapped to the pre-big bang solution

$$
a=(-t)^{-1 / 2}, \quad \phi=-3 \ln (-t), \quad p=-\rho / 3, \quad t<0
$$

(see also Refs. $[18,19]$ for other, less trivial examples).

This natural association of pre- and post-big bang solutions, which always come in pair from the lowest-order string cosmology equations, is impossible in the standard cosmological context based on the Einstein equations: in that case there is in fact no field playing the role of the dilaton, and the above duality symmetries cannot be implemented. The challenge is, of course, to formulate a complete and consistent string cosmology scenario in which the two duality-related solutions are smoothly joined, in the context of a background satisfying (probably only asymptotically) the self-dual condition $a(t)=a^{-1}(-t)$. We shall assume in the rest of the paper that such a smooth transition (impossible to lowest order) can be successfully implemented when higher-order corrections are included, and we shall discuss some phenomenological aspects of the emerging cosmological scenario.

\subsection{Kinematical aspects}

From a kinematical point of view, the epoch of pre-big bang evolution can be

invariantly characterized as a phase of shrinking event horizonst. This means that the scale factor $a(t)$, for an isotropic phase of pre-big bang evolution, can be parametrized in cosmic time as

$$
a(t)=(-t)^{\beta}, \quad \beta<1, \quad t<0,
$$

where the condition $\beta<1$ is required for the convergence of the integral defining the comoving size of the event horizon:

$$
\int_{t}^{0} \frac{d t^{\prime}}{a\left(t^{\prime}\right)}<\infty \Rightarrow \beta<1
$$

This condition can be satisfied in two ways: 
- $\beta<0 \Rightarrow \dot{a}>0, \ddot{a}>0, \dot{H}>0$,

which corresponds to a metric describing a phase of accelerated expansion and growing curvature, usually called superinflation 21 (or pole inflation).

- $0<\beta<1 \Rightarrow \dot{a}<0, \ddot{a}<0, \dot{H}<0$,

which corresponds to a metric describing accelerated contraction and, again, growing curvature scale.

The first type of metric provides a representation of the pre-big bang scenario in the String frame (also called Brans-Dicke frame), in which test strings move along geodesic surfaces, the second in the more conventional Einstein frame, in which the gravi-dilaton action appears diagonalized in the standard, canonical way. The String frame was explicitly used above for presenting the effective action (2.1) and the duality transformations, the Einstein frame will be used below for discussing the amplification of perturbations. The choice of the frame is indeed a matter of convenience, as both frames give consistent and physically equivalent descriptions of the pre-big bang scenario (see Refs. [2,3] for the explicit relation between the two frames and for a discussion of their physical equivalence).

In the context of this paper, what is important is the fact that both classes of backgrounds, irrespective of the sign of $\beta$, are accelerated, and in both classes the amplification of perturbations is more efficient, at high frequency, than in a standard inflationary background. This occurs for two reasons:

- First because the curvature scale is growing and, as a consequence, the perturbation spectrum 22, 23 grows with frequency 2124 ;

- Second because the comouing amplitude of perturbations may grow in time even outside the horizon 225, instead of being frozen as in the standard scenario.

These two effects are crucial for obtaining a strong graviton background at high frequency, and will thus be discussed in some detail in the next section.

\section{Growth of perturbations}

For an accelerated background, the scale factor can be conveniently parametrized in conformal time $(\eta$, such that $d t=a d \eta)$, and in the negative time range, as

$$
a(\eta)=(-\eta)^{\alpha}, \quad \eta<0 .
$$

Positive $\alpha$ corresponds to accelerated contraction, negative $\alpha$ to accelerated expansion, $\alpha=-1$ corresponds in particular to the standard de Sitter-like inflation (see Fig. 2).

Consider for instance the canonical variable $h_{\mu \nu}$ representing tensor perturbations, in the Einstein frame, normalized to an initial vacuum fluctuation spectrum, 


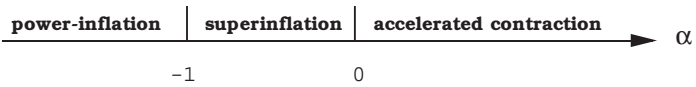

Figure 2: Different classes of accelerated backgrounds, parametrized in conformal time by the power $\alpha$.

i.e. $h_{k} \sim\left(e^{-i k \eta} / a M_{p} \sqrt{k}\right)$, for $\eta \rightarrow-\infty\left(M_{p}\right.$ is the Planck mass). The Fourier component of each polarization mode $h_{k}$ satisfies the well-known equation 22,23

$$
\left(a h_{k}\right)^{\prime \prime}+\left(k^{2}-\frac{a^{\prime \prime}}{a}\right) a h_{k}=0 .
$$

In a background of the pre-big bang type the horizon is shrinking, so that all modes are "pushed out" of the horizon, for $\eta \rightarrow 0_{-}$. For a mode whose wavelength is larger than the horizon size $(|k \eta|<<1)$, the asymptotic solution of the above perturbation equation is given by

$$
h_{k}=A_{k}+B_{k}|\eta|^{1-2 \alpha}, \quad \eta \rightarrow 0_{-}
$$

where $A_{k}$ and $B_{k}$ are integration constants. We have in general two possibilities.

- If $\alpha<1 / 2$ the first term is dominant in the asymptotic solution $(3.3)$, the comoving amplitude $h_{k}$ tends to stay constant outside the horizon, and the typical (dimensionless) perturbation amplitude $\delta_{h}(k)$ (see Appendix A) can be expressed as usual in terms of the Hubble factor at horizon crossing:

$$
\left|\delta_{h}(k)\right| \equiv k^{3 / 2}\left|h_{k}\right| \simeq\left(\frac{H}{M_{p}}\right)_{h . c .}, \quad \text { horizon crossing } \Leftrightarrow|k \eta| \sim 1 .
$$

This amplitude is constant in time. According to the horizon crossing condition, however, higher-frequency modes cross the horizon later in time, and then at higher value of the Hubble factor if the curvature scale is growing, as in our class of backgrounds. As a consequence, the amplitude of higher-frequency modes is enhanced with respect to lower frequency modes, in contrast with the usual scale-invariant (Harrison-Zeldovich) spectrum, where the amplitude is the same for all modes.

- If $\alpha>1 / 2$ the solution (3.3) is dominated by the second term, and $h_{k}$ tends to grow outside the horizon. The typical perturbation amplitude becomes time-dependent,

$$
\left|\delta_{h}(k, \eta)\right| \simeq\left(\frac{H}{M_{p}}\right)_{h . c .}|k \eta|^{1-2 \alpha}, \quad \eta \rightarrow 0_{-},
$$

and grows not only with frequency, but also with time. This second effect is not automatic, however, in any background of the pre-big bang type, but requires a fast enough contraction (in the Einstein frame) of the scale factor. 


\subsection{Formal problems}

The analysis of the perturbation spectrum, in the context of the pre-big bang scenario, is complicated in general by two types of formal problems.

The first is that, just because of this "anomalous" growth in time of the amplitude, the linear approximation tends to break down for scalar perturbations, in the standard longitudinal gauge. This problem may be solved, at least for a class of dilaton-driven backgrounds, by moving to an off-diagonal gauge 26 (also called "uniform-curvature" gauge27), in which the scalar part of the perturbed metric is parametrized by two scalar potentials, $\Phi$ and $B$, as follows

$$
d s^{2}=a^{2}\left[d \eta^{2}(1+2 \Phi)-d x_{i}^{2}-2 \partial_{i} B d x^{i} d \eta\right] .
$$

In this way the dangerous growing mode is "gauged down", enough to restore the validity of the linear approximation. For other backgrounds, however, the growth of perturbations outside the horizon may be a true physical effect, which cannot be eliminated in a convenient gauge; we have to restrict ourselves to a reduced portion of parameter space for the linear approximation to be valid.

A second problem is related to the normalization of the perturbations to the initial spectrum of the quantum fluctuations of the vacuum, in a background that is in general higher-dimensional and anisotropic. Such a normalization requires the knowledge of the so-called "normal modes" of oscillation of an anisotropic background, i.e. of the variables that diagonalize the action perturbed to second order, and that satisfy canonical commutation relations. This problem has been solved, up to now, only for spatially flat higher-dimensional backgrounds, in which the translations along internal dimensions are isometries of the full perturbed metric 2 .

In any case, even if we have to restrict ourselves to a limited class of backgrounds in which the linear approximation is valid, and the canonical normalization is known, we find that the enhanced amplification of perturbation, in the context of the prebig bang scenario, is in general associated to new and interesting phenomenological consequences.

\subsection{Phenomenological consequences}

There are two effects, in particular, worth mentioning. The first is the amplification of the vacuum fluctuations of the electromagnetic field (and of the gauge fields, in general), due to their coupling to a dynamical dilaton, according to the effective interaction

$$
e^{-\phi} \sqrt{-g} F_{\mu \nu} F^{\mu \nu}
$$

The electromagnetic field is also coupled to the metric, but in four dimensions such a coupling is conformally invariant, and there is no parametric amplification of perturbations in a conformally flat metric backgrounde9, like that of a typical inflationary model. In a string cosmology context, the amplification of the electromagnetic perturbations is a consequence of the dilaton running during the phase of pre-big bang evolution. Such an amplification may be large enough to produce the 
"seed fields" required for the origin of the galactic magnetic field 30 , and possibly also to produce the observed anisotropy 31 of the Cosmic Microwave Background $(\mathrm{CMB})$ radiation 32 .

A second important effect is the production of a relic graviton background, mych stronger, at high frequency, than predicted by the standard inflationary scenario 33 .

The present spectral energy density of such a background, in units of critical energy density $\rho_{c}$, is defined as

$$
\Omega_{G}\left(\omega, t_{0}\right)=\frac{\omega}{\rho_{c}} \frac{d \rho_{G}\left(\omega, t_{0}\right)}{d \omega}, \quad \rho_{c}\left(t_{0}\right)=\frac{3 M_{p}^{2} H_{0}^{2}}{8 \pi},
$$

where

$$
\frac{d \rho_{G}\left(\omega, t_{0}\right)}{d \omega}=2 \omega \bar{n}(\omega)\left[\frac{4 \pi \omega^{2}}{(2 \pi)^{3}}\right]
$$

and where $\bar{n}(\omega)$ is the number density of produced particles with proper energy $\omega(t)=k / a(t)$ (the factor 2 is because of the two graviton polarization modes; the factor in square brackets refers to integration in momentum space). The peak value of $\Omega_{G}$, for any model of cosmological evolution, can be conveniently referred to the present CMB energy density $\Omega_{\gamma}$ (see next section), which is a number of order 3 $10^{-4}-10^{-5}$ (the uncertainty depends on the present uncertainty about the value of $\rho_{c}$ ). In the standard inflationary scenario the energy density of the graviton background is constrained by the high degree of isotropy of the CMB radiation 31 , $(\Delta T / T)_{\gamma} \lesssim 10^{-5}$, which imposes, at all scales $\omega>10^{-16} \mathrm{~Hz}$, the bound 35.36

$$
\Omega_{G} \lesssim \Omega_{\gamma}\left(\frac{\Delta T}{T}\right)_{\gamma}^{2} \lesssim 10^{-15}
$$

In the context of the pre-big bang scenario, on the contrary, the graviton background is in general tog low, on the COBE scale $\omega_{0} \sim 10^{-18} \mathrm{~Hz}$, to be constrained by the CMB isotropy 3 en 33 . The peak value of the spectrum is simply controlled by the present value of the fundamental ratio of the string and Planck mass, which is expected 37 to be a number in the range $0.3-0.03$ :

$$
\Omega_{G} \lesssim \Omega_{\gamma}\left(\frac{M_{s}}{M_{p}}\right)^{2} \lesssim 10^{-6}
$$

This implies a possible signal, in the frequency range of present and near-future gravity wave detectors, up to nine orders of magnitude higher than predicted by the standard inflationary scenario. The next section is devoted to a detailed discussion of this possibility.

\section{The Expected Graviton Background}




\subsection{Minimal model of pre-big bang}

In order to discuss the expected graviton background we shall first consider a "minimal" model of pre-big bang scenario (the same as used in Refs. [29, 32]), in which there are only three phases: an initial phase of dilaton-driven inflation for $t<t_{s}$, a high curvature string phase for $t_{s}<t<t_{r}$, and the standard radiation-dominated evolution for $t>t_{r}$ (see also Fig. 1).

At low frequency, namely for modes with $\omega<<\omega_{s} \simeq\left(a_{s} \eta_{s}\right)^{-1}$, which cross the horizon before the beginning of the string phase, the slope of the spectrum can be computed exactly from the perturbation equation obtained from the lowenergy action, and it is found to be cubic 2633 (modulo small corrections due to a logarithmic growth in time of the comoving amplitude outside the horizon, during the dilaton-driven phase):

$$
\Omega_{G}\left(\omega, t_{0}\right) \propto\left(\frac{\omega}{\omega_{s}}\right)^{3} \ln ^{2}\left(\frac{\omega}{\omega_{s}}\right), \quad \omega<\omega_{s} .
$$

In this range of frequencies, the spectrum can easily be computed by noting that, for the given model of background, the tensor perturbation equation (3.2) reduces to a Schrödinger-like equation in conformal time22 23 :

$$
\psi_{k}^{\prime \prime}+\left[k^{2}-V(\eta)\right] \psi_{k}=0,
$$

with an effective potential ( $V=a^{\prime \prime} / a$ in the Einstein frame) whose modulus grows during the dilaton phase, keeps growing in the string phase where it reaches a maximum around the transition scale $\eta_{r}$, and rapidly approaches zero at the beginning of the radiation era (see Fig. 3).

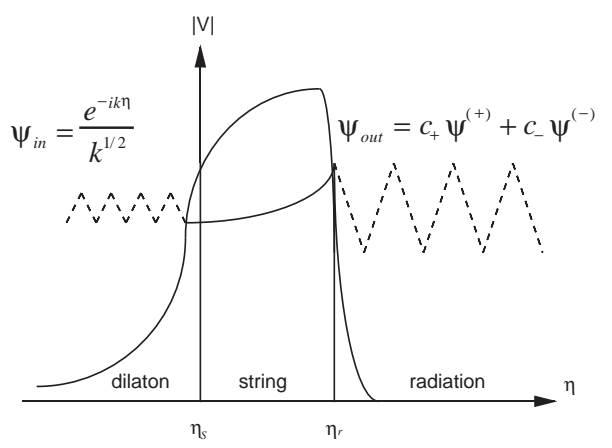

Figure 3: Parametric amplification of the perturbation mode $\psi_{k}$ due to the effective potential barrier $V=a^{\prime \prime} / a$ ("anti-tunnelling" effect).

Normalizing the solution at $\eta \rightarrow-\infty$ to an initial vacuum fluctuation spectrum, $\psi_{k} \sim e^{-i k \eta} / \sqrt{k}$, the asymptotic solution at late times in the radiation era, for $\eta \rightarrow+\infty$, is in general a linear combination of modes of positive and negative frequency with respect to the initial vacuum state:

$$
\psi_{k}=c_{+} \psi_{k}^{(+)}+c_{-} \psi_{k}^{(-)}, \quad \eta \rightarrow+\infty,
$$


where the mixing coefficients $c_{ \pm}$parametrize the unitary Bogoliubov transformation connecting the set of $\mid$ in $\rangle$ and $\mid$ out $\rangle$ annihilation and creation operators,

$$
\begin{gathered}
\left\{\psi_{\text {in }}, b_{k}, b_{k}^{\dagger}\right\} \Longrightarrow\left\{\psi_{\text {out }}, a_{k}, a_{k}^{\dagger}\right\}, \\
a_{k}=c_{+} b_{k}+c_{-}^{*} b_{-k}^{\dagger}, \quad a_{-k}^{\dagger}=c_{-} b_{k}+c_{+}^{*} b_{-k}^{\dagger} .
\end{gathered}
$$

So, even starting from the vacuum, one ends up with a final expectation number of gravitons, which is, in general, non-vanishing 33 39. 40

$$
\bar{n}_{i}=\left\langle 0\left|b^{\dagger} b\right| 0\right\rangle, \quad \bar{n}_{f}=\left\langle 0\left|a^{\dagger} a\right| 0\right\rangle=\left|c_{-}\right|^{2} \neq 0 .
$$

The Bogoliubov coefficients $c_{ \pm}(k)$ can be computed by solving the perturbation equation (4.2) in the three different regions of Fig. 3, and by matching $\psi(\eta), \psi^{\prime}(\eta)$ at $\eta=\eta_{s}$ and $\eta=\eta_{r}$. The expectation number $\bar{n}(k)=\left|c_{-}(k)\right|^{2}$ then gives the spectral energy distribution $\Omega_{G}(\omega)$ according to eqs. (3.8), (3.9).

This process of parametric amplification 22 can be seen as an "anti-tunnelling" effect for the "wave function" $\psi_{k}$. Indeed, if we look at Fig. 3 from the right to the left, and imagine to replace the time variable $\eta$ with a spacelike coordinate, we obtain the description of a scattering process in which $\psi^{(+)} \sim e^{-i k \eta}$ plays the role of a wave incident on the barrier from $+\infty, \psi^{(-)} \sim e^{+i k \eta}$ is the reflected wave, and $\psi_{\text {in }}$ is the transmitted wave,

$$
\begin{array}{ll}
\eta \rightarrow+\infty, & \psi \sim A_{i n} e^{-i k \eta}+A_{r e f} e^{i k \eta} \\
\eta \rightarrow-\infty, & \psi \sim A_{t r} e^{-i k \eta} .
\end{array}
$$

In the semiclassical approximation, which corresponds in our case to the limit of a very large number of produced particles, $\bar{n}_{f}>>1$, the reflection coefficient $R=$ $\left|A_{\text {ref }}\right|^{2} /\left|A_{\text {in }}\right|^{2}$ is approximately 1 , and the Bogoliubov coefficient $\left|c_{-}\right|^{2}$ becomes the inverse of the tunnelling coefficient $T=\left|A_{t r}\right|^{2} /\left|A_{\text {in }}\right|^{2}$ :

$$
\left|c_{-}\right|^{2}=\frac{\left|A_{r e f}\right|^{2}}{\left|A_{t r}\right|^{2}}=\frac{R}{T} \simeq \frac{1}{T} .
$$

Hence the name anti-tunnelling.

At high frequencies, namely for modes with $\omega>>\omega_{s}$, crossing the horizon (or "hitting" the effective potential barrier) during the string phase, the exact slope of the spectrum is at present unknown. In fact, even assuming that the backorgund curvature stays constant, $H \simeq M_{s}$ (in string units) during the string phase 30.33 , we do not know the corresponding evolution of the dilaton background. Also, and most important, we do not know the exact form of the perturbation equation, because of possible higher derivatives corrections due to higher orders in the $\alpha^{\prime}$ expansion (eventually, also higher-loop contributions cannot be excluded, a priori).

It is important to stress, however, that the latter problem can at most affect the amplitude, but not the slope (4.1) of the "dilatonic" branch of the spectrum. In fact, a transverse and traceless mode of constant amplitude is always a solution 
of a linearized and covariant perturbation equation, to all orders in the background curvature 41 . This preserves the spectral distribution of modes whose amplitude is frozen already during the dilaton-driven phase.

Concerning the former problem, we can parametrize our ignorance of the background evolution with a power-like behaviour of the dilaton background 30 [33 (see also next section). By using the low-energy perturbation equation, we can thus predict for the graviton spectrum a slope that is at most cubic, but in general flatter than at lower frequencies. Interestingly enough, this property is valid for the exact solution of the low energy perturbation equation 41 , to all orders in the $k$ expansion (3.3), and for any given time-dependent background, not only of the power-law type. Since this result is a consequence of the duality symmetry characterizing the low-energy evolution of perturbations 42 , one might argue that a flatter slope at higher frequency is in general to be expected, provided the duality symmetry is not spoiled by higher derivatives and higher-loop corrections.

What we can do, also, irrespective of a detailed knowledge of the background kinematics, is to provide an accurate estimate of the present value of the end point of the spectrum, namely of the maximal frequency undergoing parametric amplification 43 . This frequency is of the same order as the frequency corresponding to the production of one graviton per polarization and per unit phase-space volume (it is known that, for higher frequencies, graviton production is exponentially suppressed29.39). To this aim we rescale such a frequency, which we call $\omega_{1}(t)$, to the beginning of the radiation era, i.e. $\omega_{1}\left(t_{0}\right)=\omega_{1}\left(t_{r}\right) a_{r} / a_{0}$, where by definition

$$
H_{r}^{2}=\frac{8 \pi}{3 M_{p}^{2}} \frac{\pi^{2} N_{r}}{30} T_{r}^{4}
$$

( $N_{r}$ is the total effective number of massless degrees of freedom in thermal equilibrium at $\left.t=t_{r}\right)$. Also, we rescale $T_{r}$ to the present $\mathrm{CMB}$ temperature $T_{0}=2.7 \mathrm{~K}$, by taking into account a possible production of radiation due to some reheating process occurring well below the string scale. The effect of such processes can be parametrized by the associate fraction of present thermal entropy density, $\delta s$, as

$$
s_{0} \equiv \frac{2 \pi^{2}}{45} n_{0}\left(a_{0} T_{0}\right)^{3}=\frac{2 \pi^{2}}{45} n_{r}\left(a_{r} T_{r}\right)^{3}+s_{0} \delta s,
$$

where $n_{0}$ and $n_{r}$ are the number of species contributing (with their own statistical weight) to the thermal entropy at $t_{0}$ and $t_{r}$, respectively $\left(n_{0}=3.91\right.$, see for instance Ref. [33]).

In a string cosmology context, the amplification of tensor perturbations is always accompanied by a copious production of gauge bosons (according to eq. (3.7)) and other ultrarelativistic particles, which are expected to thermalize rapidly, unlike gravitons and dilatons, which interact only gravitationally 44.45 . For the formulation of a consistent scenario in which the energy density of such particles, produced from the vacuum, becomes critical and start dominating the background evolution at $t=t_{r}$, we shall assume that both $H_{r}$ and $\omega_{1}\left(t_{r}\right)$ are of the same order as the string 
scale at $t_{r}$, i.e. $H_{r} \simeq M_{s}\left(t_{r}\right) \simeq \omega_{1}\left(t_{r}\right)$. In that case we obtain, from eqs. (4.9) and (4.10),

$$
\omega_{1}\left(t_{0}\right) \simeq 1.1 T_{0}\left[\frac{M_{s}\left(t_{r}\right)}{M_{p}}\right]^{1 / 2}\left(\frac{10^{3}}{n_{r}}\right)^{1 / 12}(1-\delta s)^{1 / 3}
$$

where we have used the fact that $n_{r} \simeq N_{r}$, and that $N_{r}$ is expected to be a number of order $10^{2}-10^{3}$.

On the other hand, the fact that the radiation temperature cannot exceed the string mass scale in a string cosmology context, $T_{r} \lesssim M_{s}\left(t_{r}\right)$, implies that the total energy density of the quantum fluctuations (equal to $\rho_{r}$, at $t=t_{r}$ ) is dominated by the energy density $\rho_{1}$ corresponding to the end-point frequency $\omega_{1}$, i.e. $\rho_{1}=\omega_{1}^{4} / \pi^{2}$. In fact,

$$
\frac{\rho_{r}}{N_{r} \omega_{1}^{4}\left(t_{r}\right)} \simeq \frac{\rho_{r}}{N_{r} M_{s}^{4}\left(t_{r}\right)}=\frac{\pi^{2}}{30} \frac{T_{r}^{4}}{M_{s}^{4}\left(t_{r}\right)} \lesssim 1 .
$$

In addition, the fact that $\rho_{r} \simeq N_{r} M_{s}^{4}$ is critical at $t=t_{r}$ implies that the string scale is already quite close to its present value, as $M_{p} / M_{s}\left(t_{r}\right) \simeq N_{r}^{1 / 2} \sim 10-30$. Consequently, the peak of the graviton background must be of the same order as the end-point value $\Omega_{G}\left(\omega_{1}, t_{0}\right)=\omega_{1}\left(t_{0}\right) / \pi^{2} \rho_{c}\left(t_{0}\right)$, with $M_{s}$ fixed by a dilaton expectation value already in its present range.

So, the spectral graviton distribution has to be on the average non-decreasing from $\omega_{s}$ to $\omega_{1}$, with an upper bound that can be written in critical units as 43

$$
\begin{aligned}
\Omega_{G}\left(\omega_{1}, t_{0}\right) & \simeq 2.6 \Omega_{\gamma}\left(t_{0}\right)\left(\frac{M_{s}}{M_{p}}\right)^{2}\left(\frac{10^{3}}{n_{r}}\right)^{1 / 3}(1-\delta s)^{4 / 3} \\
& \simeq 7 \times 10^{-5} h_{100}^{-2}\left(\frac{M_{s}}{M_{p}}\right)^{2}\left(\frac{10^{3}}{n_{r}}\right)^{1 / 3}(1-\delta s)^{4 / 3},
\end{aligned}
$$

where $h_{100}=H_{0} /\left(100 \mathrm{~km} \mathrm{sec}^{-1} \mathrm{Mpc}^{-1}\right)$. Note that this peak value can be integrated from $\omega_{1}$ down to the Hertz scale without contradicting the bound following from the standard nucleosynthesis analysis 34 ,

$$
h_{100}^{2} \int \Omega_{G}\left(\omega, t_{0}\right) d \ln \omega \lesssim 0.5 \times 10^{-5} .
$$

The above situation is illustrated in Fig. 4, which shows the maximum allowed graviton energy density for the case $n_{r}=10^{3}$, in the $\mathrm{Hz}$ to $\mathrm{GHz}$ range. For any given $\delta s$, the uncertainty of the end point (4.11) and of the peak (4.13) is mainly due to the present theoretical uncertainty of the fundamental ratio $M_{s} / M_{p}$, which has been chosen, for the illustrative purpose of Fig. 4, to range from 0.1 to 0.01 . This uncertainty is represented by the shaded boxes of Fig. 4. To the left of the end point the spectrum can be at most flat, so that in the absence of significant reheating at scales much lower than $t_{r}$, the expected maximal strength of the graviton background should lie within the dashed lines in the band labelled by $\delta s=0$.

A non-zero $\delta s$ would instead imply that the radiation that becomes dominant at the end of the string phase is only a fraction of the thermal radiation that we observe 
today, with a consequent dilution of the graviton background (produced at the string scale) with respect to the total present radiation energy density. However, we can see from the picture that even if $99 \%$ of the present entropy would be produced during the latest stage of evolution, the intensity of the relic graviton background would stay well above the full line labelled "de Sitter" in Fig. 4, which represents the most optimistic prediction of the standard inflationary scenarid 36 , in this range of frequency.

Also shown in the picture are three lines of constant sensitivity of a gravity wave detector, $S_{h}^{1 / 2}=10^{-23}, 10^{-25}, 10^{-27} \mathrm{~Hz}^{-1 / 2}$, given in terms of the spectral amplitude $S_{h}^{1 / 2}$, related to the spectral energy density $\Omega_{G}$ by

$$
S_{h}(\nu)=\frac{3 H_{0}^{2}}{4 \pi^{2} \nu^{3}} \Omega_{G}(\nu), \quad \nu=\omega / 2 \pi,
$$

(see Appendix B). As clearly shown in Fig. 4, a sensitivity of $10^{-25}$ in the $\mathrm{kHz}$ range (which is a typical range for present, Earth-based detectors) would already be enough to enter the region where we expect a signal, thus constraining (even with a negative result) the parameters of our class of string cosmology models 43 .

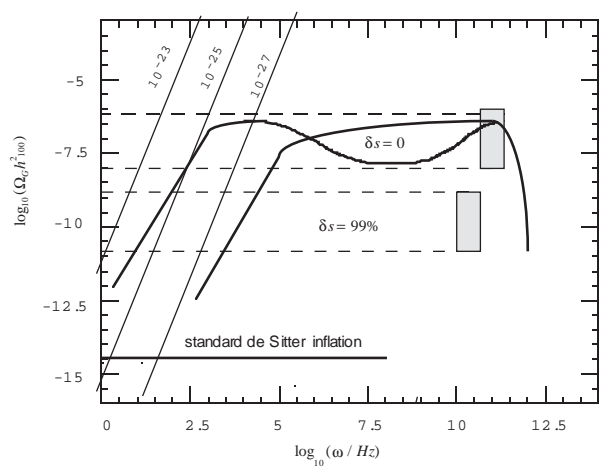

Figure 4: Position of the peak and of the end point of the relic graviton spectrum for a typical pre-big bang scenario.

In fact, any spectrum with a shape that reaches the end point, growing not faster than $\omega^{3}$, is in principle allowed in this context, as the two examples shown by the thick curves in Fig. 4. The slope has to be cubic at low enough frequencies, for modes crossing the horizon in the dilaton-driven phase. At higher frequencies the spectrum has to be on the average non-decreasing. However, it could also be non-monotonic, if $\dot{\phi}$ does not follow exactly, during the string phase, the constant behaviour of the spacetime curvature, but decreases or undergoes small oscillations around the string scale (see the next section). Interestingly enough,, an oscillating spectrum at high frequency seems to be possible even for a monotonic evolution of the dilaton background 46 .

Finally, it may be interesting to point out that for a long enough string phase it seems possible, in principle, to have a spectrum that is normalized to the end point 
value at high frequency, and which is nevertheless strong enough at the COBE scale to be significantly constrained by the present data on the CMB anisotropy.

Suppose in fact that all scales inside our present horizon $\left(\gtrsim \omega_{0} \sim 10^{-18} \mathrm{~Hz}\right)$ crossed the horizon during the high curvature string phase, and let us parametrize the present (unknown) distribution of tensor perturbations with a spectral index $n$, such that $n=1$ corresponds to the flat, scale-invariant spectrum. For all scales reentering the horizon after the time of matter-radiation equilibrium, $\omega<\omega_{e q} \sim$ $10^{-16} \mathrm{~Hz}$, there is an additional effect of parametric amplification due to the matterdominated phase, which imposes an extra $\omega^{-2}$-dependence on the spectrum 39.4 . By choosing, from eqs. (4.11) and (4.13), the typical values $\omega_{1} \sim 10^{11} \mathrm{~Hz}$ and $\Omega_{G}\left(\omega_{1}\right) \sim$ $10^{-6}$ for illustrative purpose, we then have the possible normalized distribution

$$
\begin{aligned}
\Omega_{G}\left(\omega_{1}, t_{0}\right) & =10^{-6}\left(\frac{\omega}{\omega_{1}}\right)^{n-1}, & & \omega_{e q}<\omega<\omega_{1} \\
& =10^{-6}\left(\frac{\omega}{\omega_{1}}\right)^{n-1}\left(\frac{\omega_{e q}}{\omega}\right)^{2}, & & \omega_{0}<\omega<\omega_{e q} .
\end{aligned}
$$

For the dilatonic branch of the spectrum the spectral index is $n=4$, and $\Omega_{G}$ is consequently negligible at the present horizon scale $\omega_{0}$. For the string branch of the spectrum, however, $n$ is unknown, and can be bounded from below by imposing the COBE normalization, which implie $35 \Omega_{G}\left(\omega_{0}, t_{0}\right) \sim 10^{-10}$. This condition is perfectly compatible with the end-point normalization, and gives $n=37 / 29 \simeq 1.27$. The corresponding spectral behaviour in the range $\omega_{0}<\omega<\omega_{e q}$, namely $\Omega_{G} \sim$ $\omega^{-1.73}$, is not flat enough to match the observed CMB anisotropy (a gravity wave interpretation of such an isotropy is problematic also in the standard inflationary context $(49)$. However, for $\omega>\omega_{e q}$, the resulting, slightly growing, "blue" spectrum is well consistent with the bound obtained from pulsar timing data $48, \Omega_{G}\left(\omega_{p}\right) \lesssim 10^{-8}$ at $\omega_{p} \sim 10^{-8} \mathrm{~Hz}$, as illustrated in Fig. 5 .

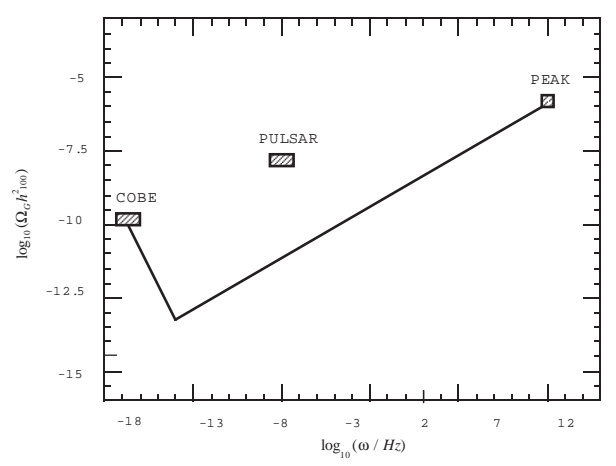

Figure 5: Example of possible graviton spectrum which has the correct peak normalization at high frequency, and that satisfies also the COBE normalization at the frequency scale of our present horizon.

Note that, by assuming the normalization at the COBE scale as input, since the 
end-point normalization is fixed, we may predict a spectrum with $\Omega_{G} \sim 10^{-8}$ in the $\mathrm{kHz}$ range, which seems to be accessible to the sensitivity of gravity-wave detectors of the second generation (see Section 4.5).

\subsection{A diagrammatic approach to the perturbation spectrum}

At this point, two remarks are in order. The first concerns the possibility of constraining the graviton spectrum through the associated amplification of electromagnetic fluctuations, which in our context is always expected to accompany graviton production. The second concerns the possible qualitative modification of the spectrum, in models of background more complicated than the minimal one previously considered.

A complete discussion of these points would require a detailed knowledge of the kinematics and of the higher-order corrections during the string phase. In the absence of such knowledge, I will approximate the background with a finite number of phases, each of them characterized by a different type of power-law evolution. Also, I will use the lowest-order perturbation equation, working however only in the asymptotic regimes $|k \eta|<<1$ and $|k \eta|>>1$ (also called, sometimes improperly, outside and inside the horizon), assuming that the qualitative behaviour of the asymptotic solution is not spoiled by higher-order corrections (see the previous section for arguments supporting this assumption).

Under the above assumptions, the graviton spectrum can be conveniently obtained through a sort of "diagrammatic" technique, in which one has only to specify the power (in conformal time) of the scale factor of the various phases, and the time of horizon crossing of the frequency band considered.

This technique is based on the fact that the exact solution of the perturbation equation (4.2), for a generic power-law evolution of the Einstein frame scale factor, $a \sim|\eta|^{\alpha}$, can be written in terms of the first-kind $\left(H_{\nu}^{(1)}\right)$ and second-kind $\left(H_{\nu}^{(2)}\right)$ Hankel functions, of index $\nu=|\alpha-1 / 2|$, as follows:

$$
a=|\eta|^{\alpha} \Rightarrow \psi_{k}=\left|\eta_{k}\right|^{1 / 2}\left[A_{+}(k) H_{\nu}^{(2)}(|k \eta|)+A_{-}(k) H_{\nu}^{(1)}(|k \eta|)\right], \quad \nu=|\alpha-1 / 2|
$$

( $A_{ \pm}$are integration constants). When matching the solution from a phase with power $\alpha_{2}$ to a phase with power $\alpha_{1}$, at the time $\eta=\eta_{1}$,

$$
\psi_{k}=\psi_{k}^{2}\left(A_{ \pm}^{2}, \nu_{2}\right), \quad \eta<\eta_{1} ; \quad \psi_{k}=\psi_{k}^{1}\left(A_{ \pm}^{1}, \nu_{1}\right), \quad \eta>\eta_{1}
$$

we may thus easily distinguish the two asymptotic regimes $\left|k \eta_{1}\right|<<1$ and $\left|k \eta_{1}\right|>>$ 1 , corresponding respectively to the comoving frequency of a mode that hits and one that does not hit the potential barrier of eq. (4.2), whose height at $\eta_{1}$ is $\left|V_{1}\right|^{1 / 2} \sim\left|\eta_{1}\right|^{-1}$. In the first case, by using the small argument limit of the Hankel functions 50 , the continuity of $\psi$ and $\psi^{\prime}$ gives

$$
\begin{aligned}
& \left(A_{+}^{1}+A_{-}^{1}\right)=b_{1}\left(A_{+}^{2}+A_{-}^{2}\right) x_{1}^{\nu_{2}-\nu_{1}}+b_{2}\left(A_{+}^{2}-A_{-}^{2}\right) x_{1}^{-\nu_{2}-\nu_{1}} \\
& \left(A_{+}^{1}-A_{-}^{1}\right)=b_{3}\left(A_{+}^{2}+A_{-}^{2}\right) x_{1}^{\nu_{2}+\nu_{1}}+b_{4}\left(A_{+}^{2}-A_{-}^{2}\right) x_{1}^{-\nu_{2}+\nu_{1}}
\end{aligned}
$$


where $x_{1}=\left|k \eta_{1}\right|$ and $b_{1}, b_{2}, b_{3}, b_{4}$ are complex numbers with modulus of order 1 (for $\nu=0, x^{\nu}$ has to be replaced by $\ln x$ ). In the second case, $|k \eta|>>1$, the perturbation is approximately unaffected by the background transition, and the large argument limit of the Hankel functions gives

$$
A_{+}^{1}=A_{+}^{2}, \quad A_{-}^{1}=A_{-}^{2} .
$$

Suppose now to approximate the background evolution with $n+1$ different phases, with powers $\alpha_{1}, \alpha_{2}, \ldots, \alpha_{n+1}$, and solutions $\psi_{1}, \psi_{2}, \ldots, \psi_{n+1}$ of the perturbation equations, with the corresponding Bessel index $\nu_{1}, \nu_{2}, \ldots, \nu_{n+1}$. The transitions occur, respectively, at the times $\eta_{n}<\eta_{n-1}<\ldots<\eta_{2}<\eta_{1}$, and the height of the effective potential at the transition time $\eta_{i}$ is $\left|V_{i}\right|^{1 / 2} \sim\left|\eta_{i}\right|^{-1} \equiv k_{i}$ (see Fig. 6). Normalizing the initial solution to a vacuum fluctuation spectrum,

$$
A_{+}^{n+1}=1, \quad A_{-}^{n+1}=0, \quad \psi_{n+1}=|\eta|^{1 / 2} H_{\nu_{n+1}}^{(2)}(|k \eta|),
$$

the Bogoliubov coefficient determining the expectation number of particles, appearing in the final spectral distribution $\Omega_{G}$ (see eq.(3.9) ), is then given by $c_{-}(k)=$ $A_{-}^{1}(k)$.

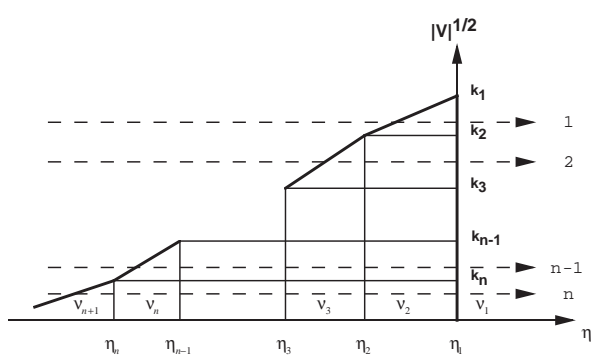

Figure 6: A background evolution parameterized by $n+1$ phases is in general associated to a spectrum with $n$ different branches. The corresponding Bogoliubov coefficients are given in eq. (4.22).

In the computation of $A_{ \pm}^{1}$ we can easily identify $n$ different branches of the spectrum, (1), (2), .., (n) (see Fig. 6), corresponding to $n$ asymptotic frequency bands, which only differ for the power characterizing the scale factor at the time of horizon crossing. By applying, step by step, the matching conditions (4.19) and (4.20), one can easily compute the Bogoliubov coefficients $c_{1}, c_{2}, \ldots, c_{n}$, for the frequency bands that are affected, respectively, by $1,2, \ldots, n$ background transitions. Neglecting numerical coefficients of the order of unity, we have

$$
\begin{aligned}
\left|c_{1}\right| & \sim x_{1}^{-\nu_{1}} x_{1}^{-\nu_{2}}, \quad k_{2}<k<k_{1} \\
\left|c_{2}\right| & \sim x_{1}^{-\nu_{1}}\left(\frac{x_{1}}{x_{2}}\right)^{-\epsilon_{2} \nu_{2}} x_{2}^{-\nu_{3}}, \quad k_{3}<k<k_{2} \\
\left|c_{3}\right| & \sim x_{1}^{-\nu_{1}}\left(\frac{x_{1}}{x_{2}}\right)^{-\epsilon_{2} \nu_{2}}\left(\frac{x_{2}}{x_{3}}\right)^{-\epsilon_{3} \nu_{3}} x_{3}^{-\nu_{4}}, \quad k_{4}<k<k_{3}
\end{aligned}
$$




$$
\left|c_{n}\right| \sim x_{1}^{-\nu_{1}}\left(\frac{x_{1}}{x_{2}}\right)^{-\epsilon_{2} \nu_{2}}\left(\frac{x_{2}}{x_{3}}\right)^{-\epsilon_{3} \nu_{3}} \ldots \ldots\left(\frac{x_{n-1}}{x_{x}}\right)^{-\epsilon_{n} \nu_{n}} x_{n}^{-\nu_{n+1}}, k<k_{n}
$$

where

$$
x_{i}=\left|k \eta_{i}\right|, \quad \epsilon_{i}=\operatorname{sign} \ln \left(\frac{x_{i}}{x_{i-1}}\right), \quad i=1,2, \ldots, n .
$$

The $\epsilon_{i}$ coefficients have been inserted to take into account the possibility of a nonmonotonic potential (unlike that of Fig. 6), since for $x_{i}<x_{i-1}$ the ratio $x_{i-1} / x_{i}$ enters the Bogoliubov coefficients with the opposite power. Note also that a phase with vanishing effective potential, like radiation-dominated evolution, $\alpha_{i}=0$, has a Bessel index $\nu_{i}=1 / 2$.

As a simple application of the general prescription (4.22), let us write down the Bogoliubov coefficients for a background characterized by four different phases and by a non-monotonic effective potential, which has a first break at $\eta_{s}$, a peak at $\eta_{1}$, and which becomes radiation-dominated at $\eta_{r}$, with $x_{s}>x_{r}>x_{1}$ (see Fig. 7). The Bessel indices are, respectively, $\nu_{0}, \nu_{s}, \nu_{1}$ and $\nu_{r}=1 / 2$. The graviton spectrum is characterized by three branches, differing by the number of background transitions by which a given mode is affected. The corresponding Bogoliubov coefficients can be immediately deduced from the diagram of Fig. 7, by applying the general prescription (4.22):

$$
\begin{array}{rlrl}
\left|c_{1}\right| & \sim x_{1}^{-\nu_{1}} x_{1}^{-\nu_{s}}, & k_{r}<k<k_{1} \\
\left|c_{2}\right| & \sim x_{r}^{-1 / 2}\left(\frac{x_{r}}{x_{1}}\right)^{\nu_{1}} x_{1}^{-\nu_{s}}, \quad k_{s}<k<k_{r} \\
\left|c_{3}\right| & \sim x_{r}^{-1 / 2}\left(\frac{x_{r}}{x_{1}}\right)^{\nu_{1}}\left(\frac{x_{1}}{x_{s}}\right)^{-\nu_{s}} x_{s}^{-\nu_{0}}, \quad k<k_{s}
\end{array}
$$

A similar type of background will be discussed in Section 4.4.

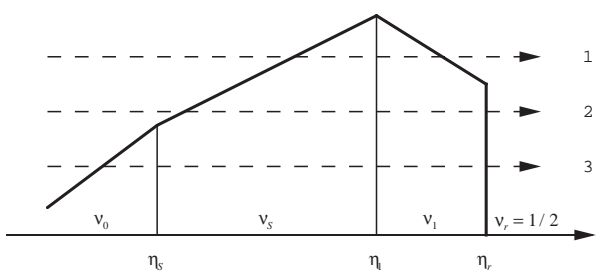

Figure 7: Example of background with non-monotonic potential. .

As discussed in the previous section, the square of the Bogoliubov coefficient determines the expectation number of the produced gravitons and the spectral distribution $\Omega_{G}$, according to eqs. (3.8) and (3.9). In critical units,

$$
\Omega_{G}(\omega, t)=\frac{\omega}{\rho_{c}} \frac{d \rho}{d \omega}=\frac{8 \omega^{4}}{3 \pi M_{p}^{2} H^{2}(t)}\left|c_{-}\right|^{2} .
$$


By expressing all ratios in terms of proper frequencies, $x_{i} / x_{j} \equiv\left|k \eta_{i}\right| /\left|k \eta_{j}\right| \equiv$ $k_{j} / k_{i}=\omega_{j} / \omega_{i}$, and referring the spectrum to the proper frequency of the final transition, $\omega_{1}(t)=k_{1} / a(t) \sim H_{1} a_{1} / a$, we easily obtain, from eqs. (4.22) and (4.25), the spectral distribution $\Omega(\omega)$ corresponding to the $n$ branches of Fig. 6 :

$$
\begin{aligned}
& \Omega_{1} \sim\left(\frac{H_{1}}{M_{p}}\right)^{2}\left(\frac{H_{1}}{H}\right)^{2}\left(\frac{a_{1}}{a}\right)^{4}\left(\frac{\omega}{\omega_{1}}\right)^{4-2 \nu_{1}-2 \nu_{2}}, \quad \omega_{2}<\omega<\omega_{1} \\
& \Omega_{2} \sim\left(\frac{H_{1}}{M_{p}}\right)^{2}\left(\frac{H_{1}}{H}\right)^{2}\left(\frac{a_{1}}{a}\right)^{4}\left(\frac{\omega}{\omega_{1}}\right)^{4-2 \nu_{1}-2 \nu_{3}}\left(\frac{\omega_{2}}{\omega_{1}}\right)^{-2 \epsilon_{2} \nu_{2}+2 \nu_{3}}, \quad \omega_{3}<\omega<\omega_{2} \\
& \Omega_{3} \sim\left(\frac{H_{1}}{M_{p}}\right)^{2}\left(\frac{H_{1}}{H}\right)^{2}\left(\frac{a_{1}}{a}\right)^{4}\left(\frac{\omega}{\omega_{1}}\right)^{4-2 \nu_{1}-2 \nu_{4}}\left(\frac{\omega_{2}}{\omega_{1}}\right)^{-2 \epsilon_{2} \nu_{2}}\left(\frac{\omega_{3}}{\omega_{2}}\right)^{-2 \epsilon_{3} \nu_{3}}\left(\frac{\omega_{1}}{\omega_{3}}\right)^{-2 \nu_{4}} \text {, } \\
& \omega_{4}<\omega<\omega_{3}
\end{aligned}
$$

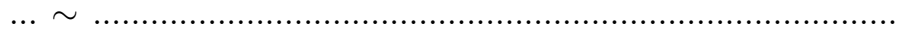

$$
\begin{aligned}
& \Omega_{n} \sim\left(\frac{H_{1}}{M_{p}}\right)^{2}\left(\frac{H_{1}}{H}\right)^{2}\left(\frac{a_{1}}{a}\right)^{4}\left(\frac{\omega}{\omega_{1}}\right)^{4-2 \nu_{1}-2 \nu_{n+1}}\left(\frac{\omega_{2}}{\omega_{1}}\right)^{-2 \epsilon_{2} \nu_{2}}\left(\frac{\omega_{3}}{\omega_{2}}\right)^{-2 \epsilon_{3} \nu_{3}} \ldots \\
& \ldots \quad\left(\frac{\omega_{n}}{\omega_{n-1}}\right)^{-2 \epsilon_{n} \nu_{n}}\left(\frac{\omega_{1}}{\omega_{n}}\right)^{-2 \nu_{n+1}}, \quad \quad \omega<\omega_{n} .
\end{aligned}
$$

It is important to point out that the slope of the spectrum, for a given branch $\Omega_{i}$, is sensitive to the particular time evolution of the background only when hitting and when leaving the barrier: in fact, $\Omega_{i}(\omega) \sim \omega^{4-2 \nu_{1}-2 \nu_{i+1}}$. Note also that, if we wish to refer the spectrum to a frequency $\omega_{k}$ other than $\omega_{1}$ (for instance to the peak frequency, which may be different from $\omega_{1}$ if the potential is non-monotonic), we have to simply multiply the $i$-th branch of the spectrum by $\left(\omega_{k} / \omega_{1}\right)^{4-2 \nu_{1}-2 \nu_{i+1}}$.

Let us now apply the general prescription (4.26) to a typical pre-big bang scenario, in which the background evolves from an initial dilaton-driven phase with $\nu_{n+1}=0$. Eventually, the background becomes radiation-dominated, so that $\nu_{1}=1 / 2$, and the ratio

$$
\left(\frac{H_{1}}{H}\right)^{2}\left(\frac{a_{1}}{a}\right)^{4}=\Omega_{r}(t)
$$

gives the radiation energy density, in critical units, rescaled to a time $t>t_{1}$. The various phases from $\eta_{n}$ to $\eta_{1}$ correspond to possible types of background during the string era, and the ratio

$$
g_{1}=H_{1} / M_{p}
$$

is taken to be of the same order as the present value of the fundamental ratio between string and Planck mass, $g_{1} \sim M_{s} / M_{p} \sim 0.3-0.03$. Let us assume, also, that the evolution of the metric background, in the Einstein frame, is always accelerated from $\eta_{n}$ to $\eta_{1}$, so that the potential $|V(\eta)|$ is non-decreasing, $\omega_{1}$ is also the maximal amplified frequency, and $\epsilon_{i}=1$ for all the transitions. The spectral distribution (4.26) for the $i$-th band of frequency, crossing the horizon during the string phase 
$(i<n)$, and affected by $i$ background transitions, can thus be written

$$
\begin{aligned}
\Omega_{i}(\omega) \sim & g_{1}^{2} \Omega_{r}(t)\left(\frac{\omega}{\omega_{1}}\right)^{3-2 \nu_{i+1}}\left(\frac{\omega_{2}}{\omega_{1}}\right)^{-2 \nu_{2}}\left(\frac{\omega_{3}}{\omega_{2}}\right)^{-2 \nu_{3}} \ldots \\
& \ldots .\left(\frac{\omega_{i}}{\omega_{i-1}}\right)^{-2 \nu_{i}}\left(\frac{\omega_{1}}{\omega_{i}}\right)^{-2 \nu_{i+1}}, \quad \omega_{i+1}<\omega<\omega_{i} .
\end{aligned}
$$

As anticipated in the previous section, since $\nu_{i+1} \geq 0$, this spectrum is characterized by a maximal slope $\Omega \sim \omega^{3}$ (which is the same slope as that of the frequency band crossing the horizon in the dilaton-driven phase, modulo log corrections). Also, since $\omega_{n}<\omega_{n-1}<\ldots<\omega_{2}<\omega_{1}$, the peak of the spectrum, $g_{1}^{2} \Omega_{r}$, is reached at $\omega=\omega_{1}$, so that the spectral distribution has to be on the average non-decreasing from $\omega_{n}$ to $\omega_{1}$, i.e. $\Omega_{i} \leq \Omega_{1}$. However, the spectrum could be non-monotonic during the string phase (in spite of the monotonicity of the potential). Consider in fact the ratio

$$
\frac{\Omega_{i}\left(\omega_{i}\right)}{\Omega_{i-1}\left(\omega_{i-1}\right)}=\left(\frac{\omega_{i}}{\omega_{i-1}}\right)^{3-2 \nu_{i}}
$$

As $\omega_{i}<\omega_{i-1}$, the spectrum may be decreasing in the frequency band $\omega_{i}<\omega<\omega_{i-1}$, provided $3<2 \nu_{i}=\left|2 \alpha_{i}-1\right|$. If we assume a constant curvature evolution in the String frame, the decreasing slope may be due to a phase of decreasing dilaton.

Suppose in fact that, during the string phase, there is a period in which the scale factor and the dilaton evolve in time (in the String frame) as

$$
\tilde{a}_{i}=(-\eta)^{-1}, \quad \tilde{\phi}_{i}=\gamma \ln \tilde{a}_{i}, \quad \gamma<0
$$

(corresponding to $\tilde{H}_{i}=$ const and decreasing dilaton, $\dot{\tilde{\phi}}_{i}=\gamma \tilde{H}_{i}<0$ ). Tranforming to the Einstein frame2 3 we have

$$
a_{i}=\tilde{a}_{i} e^{-\phi_{i} / 2}=(-\eta)^{-1+\gamma / 2}, \quad \phi_{i}=\tilde{\phi}_{i}=-\gamma \ln (-\eta)
$$

(conformal time is the same in the two frames). The slope of the corresponding frequency band is then decreasing, since

$$
3-2 \nu_{i}=3-\left|2 \alpha_{i}-1\right|=3+2\left(\frac{\gamma}{2}-1\right)-1=\gamma<0 .
$$

\subsection{Constraints from photon production}

In the context of the pre-big bang scenario, the time variation of the background fields that amplifies the perturbations of the metric tensor also amplifies the quantum fluctuations of the electromagnetic field 30.51. The electromagnetic perturbation equation has the same form as eq. (4.2), with the only difference that the effective potential is generated by a time-dependent dilaton field,

$$
V(\eta)=\frac{\phi^{\prime 2}}{4}-\frac{\phi^{\prime \prime}}{2}
$$


For a cosmological evolution that can be approximated by various phases, characterized by a logarithmic variation of the dilaton, $\phi_{i}=-\beta_{i} \ln |\eta|$, the electromagnetic perturbation spectrum can thus be computed with the diagrammatic technique illustrated in the previous section. The result is a spectrum with the same general structure as that of the graviton spectrum, but with different (in general) values of the Bessel indices $\nu_{i}$. The phenomenological bounds imposed on electromagnetic perturbations constrain the parameters of the cosmological background and then, indirectly, also the unknown slope of the graviton spectrum during the string phase.

In order to discuss this effect we shall work in the context of the minimal model of Section 4.1, characterized by three phases (dilaton $\rightarrow$ string $\rightarrow$ radiation), and we shall parametrize the unknown background evolution during the string phase (in the Einstein frame) with two powers $\alpha$ and $\beta$,

$$
a \sim|\eta|^{\alpha}, \quad \phi \sim-2 \beta \ln |\eta|, \quad \eta_{s}<\eta<\eta_{r} .
$$

For the dilaton-driven phase $\left(\eta<\eta_{s}\right)$ and for the radiation-dominated phase $(\eta>$ $\eta_{r}$ ) the background evolution is known 2 3, and is given respectively by

$$
\begin{aligned}
& a \sim|\eta|^{1 / 2}, \quad \phi \sim-\sqrt{3} \ln |\eta|, \quad \eta<\eta_{s}, \\
& a \sim|\eta|, \quad \phi \sim \text { const, } \quad \eta>\eta_{r} \text {. }
\end{aligned}
$$

The effective potential $|V(\eta)|$ grows monotonically for $\eta<\eta_{r}$ and goes rapidly to zero for $\eta>\eta_{r}$, both for gravitons and photons. The spectra have two branches, which can easily be deduced from the diagram of Fig. 8.

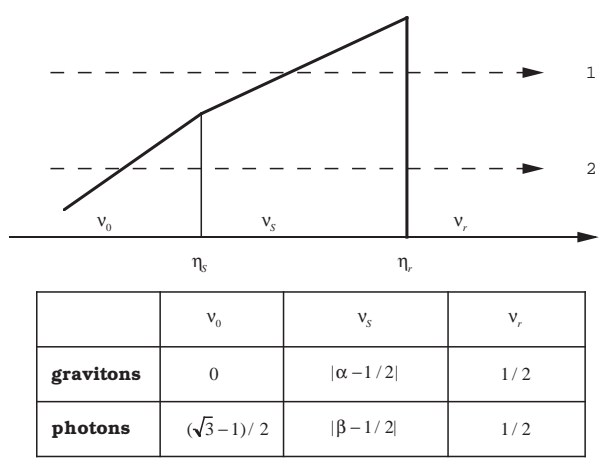

Figure 8: Diagram and Bessel indices for the graviton and photon spectrum in a minimal, two-parameter model of background.

The Bessel indices $\nu_{i}$ are fixed by the time evolution of the background, according to eqs. (4.35) and 4.36 The two parameters $\alpha$ and $\beta$ are not independent, however, if we require 303 (as seems natural) that, during the string phase, the curvature remains controlled by the string scale $M_{s}=\lambda_{s}^{-1}$. This implies, in the Einstein frame 4 ,

$$
\left|\frac{H_{s}}{H_{1}}\right| \simeq\left|\frac{\eta_{r}}{\eta_{s}}\right|^{1+\alpha} \simeq \frac{g_{s}}{g_{r}} \simeq\left|\frac{\eta_{r}}{\eta_{s}}\right|^{\beta}, \quad g_{s}=e^{\phi_{s} / 2}, g_{r}=e^{\phi_{r} / 2},
$$


from which

$$
1+\alpha \simeq \beta \simeq-\frac{\log \left(g_{s} / g_{r}\right)}{\log \left|\eta_{s} / \eta_{r}\right|}
$$

Defining the convenient parameters

$$
\begin{aligned}
& x=\log _{10}\left|\eta_{s} / \eta_{r}\right|=\log _{10} z_{s}, \\
& y=\log _{10}\left(g_{s} / g_{r}\right)=\log _{10} \frac{H_{s} / M_{p}}{H_{r} / M_{p}},
\end{aligned}
$$

related respectively to the duration of the string phase, $z_{s}=\left|\eta_{s} / \eta_{r}\right|$, and to the corresponding shift of the dilaton coupling, $g_{s} / g_{r}$, we obtain

$$
\alpha-\frac{1}{2}=\frac{3}{2}+\frac{y}{x}, \quad \beta-\frac{1}{2}=\frac{1}{2}+\frac{y}{x}, \quad \frac{\omega_{r}}{\omega_{s}}=\frac{H_{r} a_{r}}{H_{s} a_{s}} \simeq\left|\frac{\eta_{s}}{\eta_{r}}\right|=10^{x} .
$$

By applying the rules of the previous section we can now parametrize the spectra in terms of $x$ and $y$. For the two branches of the graviton spectrum we obtain, from Fig. 8,

$$
\begin{array}{rlrl}
\Omega_{G} & \sim g_{1}^{2} \Omega_{r}(t)\left(\frac{\omega}{\omega_{1}}\right)^{3-|3+2 y / x|}, \quad 10^{-x}<\frac{\omega}{\omega_{1}}<1 \\
& \sim g_{1}^{2} \Omega_{r}(t)\left(\frac{\omega}{\omega_{1}}\right)^{3} 10^{|3 x+2 y|}, & \frac{\omega}{\omega_{1}}<10^{-x}
\end{array}
$$

(we have chosen to refer all frequencies to the maximal amplified one, $\omega_{1}$ ). With the same rules we obtain the two branches of the electromagnetic spectrum,

$$
\begin{aligned}
\Omega_{e m} & \sim g_{1}^{2} \Omega_{r}(t)\left(\frac{\omega}{\omega_{1}}\right)^{3-|1+2 y / x|}, \quad 10^{-x}<\frac{\omega}{\omega_{1}}<1 \\
& \sim g_{1}^{2} \Omega_{r}(t)\left(\frac{\omega}{\omega_{1}}\right)^{4-\sqrt{3}} 10^{x(1-\sqrt{3})+|x+2 y|}, \quad \frac{\omega}{\omega_{1}}<10^{-x} .
\end{aligned}
$$

The back reaction of the electromagnetic perturbations on the geometry turns out to be negligible, consistently with the use of a homogeneous and isotropic background, provided we impose on the two-dimensional parameter space of our model the condition $30 y \gtrsim-2 x$. Also, the amplified perturbations are large enough to act as "seeds" for the galactic magnetic field, provided52 $\Omega_{G}\left(10^{-14} \mathrm{~Hz}\right) \gtrsim 10^{-34} \Omega_{r}$. These two conditions define an allowed region in the $\{x, y\}$ plane, and constrain the possible slope of the string branch of the graviton spectrum.

The first condition, by itself, does not provide significant constraints. When combined with the second, however, we obtain the constraint

$$
2<3-\left|3+2 \frac{y}{x}\right|<4,
$$

which for the graviton spectrum (4.41) defines the allowed region shown in Fig. 9 (where I have used $g_{1}=0.1$ and $\omega_{1}=10^{11} \mathrm{~Hz}$ ). The bold dashed line in Fig. 9 
represents the prediction for the graviton spectrum for the case that the amplified electromagnetic perturbations are strong enough, on a large scale, to contribute in a significant way to the observed CMB anisotropy 32 . This possibility would require indeed a flat enough string branch of the electromagnetic spectrum, $\Omega_{e m} \sim \omega^{\epsilon}$, $0<\epsilon<1$, and could be implemented consistently with other phenomenological constraints only for a background in which the comoving amplitude of perturbations grows in time also outside the horizon 32 . In terms of our parameters this gives the conditions $y>-x / 2$ and $y / x=1+\epsilon$ and implies, for the string branch of the spectrum, a slope $\Omega_{G} \sim \omega^{2+\epsilon}$.

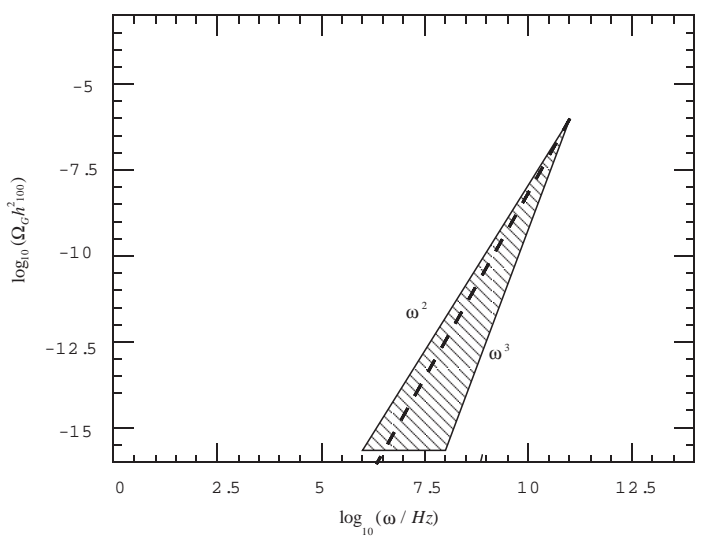

Figure 9: The shaded area shows the allowed region for the graviton spectrum, in a model in which the electromagnetic fluctuations are amplified enough to act as "seeds" for the galactic magnetic field.

It is important to note that the allowed region of Fig. 9 corresponds to a very small energy density of the relic graviton background in the $\mathrm{kHz}$ range, $\Omega_{G} \lesssim 10^{-21}$; this is certainly outside the sensitivity of gravity wave detectors now existing or planned (see Section 4.5). A possible future detection of a relic graviton background in the $\mathrm{kHz}$ range could thus be used to exclude, in our cosmological scenario, a primordial origin of the seed fields and a possible electromagnetic origin of the CMB anisotropy (at least in the context of a minimal model, and in the range of validity of the low-energy perturbation equation). Conversely, if we believe that the "dilatonic" amplification of the electromagnetic fluctuations is efficient enough to be responsible for the above effects, we should expect a graviton background outside the detector sensitivities, in the $\mathrm{kHz}$ range. This may provide strong motivations for developing detectors with improved sensitivities in the very high frequency range $(\sim \mathrm{GHz})$, near the end point of the spectrum, where the background is expected to reach the peak intensity. At lower frequencies, the absence of a signal at the sensitivity level of $10^{-6}$ (or better) in $\Omega_{G}$ would nevertheless be important, since it would support, indirectly, a primordial dilatonic origin of the cosmic magnetic fields. 


\subsection{Non-minimal models}

In the minimal model of pre-big bang assumed in the previous Sections, the end of the high curvature string phase was nearly coincident with the beginning of the radiation-dominated, constant dilaton phase. It is not impossible, however, to imagine more complex models of backgrounds, in which the dilaton is still growing while the curvature starts decreasing and where the radiation era with frozen dilaton starts at much later times, when the curvature scale is much below the string scale. An example of such a background is illustrated in Fig. 10 (the minimal model corresponds to the particular case $\eta_{r} \sim \eta_{1}$, and $g_{1} \sim 1$ ).

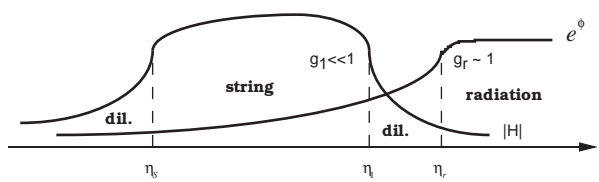

Figure 10: Non-minimal model of pre-big bang background.

It is conceivable, in fact, that the combined action of loops and $\alpha^{\prime}$ corrections may stop the growth of the curvature and may induce a change of branch of the solution, while the string coupling remains growingen. Only later could the dilaton be attracted to a fixed point of the non-perturbative potential. In that context, however, the amplified vacuum fluctuations are not expected to become naturally dominant, and the radiation should arise from other sources (for instance, coherent dilaton oscillations).

If we accept a model like that of Fig. 10, the non-vanishing part of the effective potential appearing in the perturbation equation (4.2) is no longer monotonic. Consequently, high-frequency modes may reenter the horizon (or leave the potential barrier) before the beginning of the radiation era, i.e. for $\eta_{1}<\eta<\eta_{r}$. This modifies the slope of the spectrum in the high frequency sector. For the background of Fig. 10, in particular, the spectrum has three branches, whose slope depends on the relative duration of the string phase and of the new phase inserted before the radiation era. If $\left|\eta_{r} / \eta_{1}\right|<\left|\eta_{s} / \eta_{1}\right|$, i.e. $\omega_{r}>\omega_{s}$, the spectrum corresponds to the diagram of Fig. 7. If $\omega_{r}<\omega_{s}$ the three branches of the spectrum are instead obtained from the diagram of Fig. 11, where I have supposed that the decelerated phase from $\eta_{1}$ to $\eta_{r}>0$ is again a dilaton-driven solution of the lowest order effective action, such that

$$
a \sim \eta^{1 / 2}, \quad e^{\phi} \sim \eta^{\sqrt{3}}, \quad\left(\frac{H_{1}}{H_{r}}\right) \simeq\left(\frac{g_{r}}{g_{1}}\right)^{\sqrt{3}} \simeq\left(\frac{a_{r}}{a_{1}}\right)^{3}, \quad \eta>0 .
$$

By referring the spectrum to the maximal amplified frequency $\omega_{1}$, and applying the rules of Sect. 4.2, we obtain from Fig. 11

$$
\Omega_{1} \sim g_{1}^{2}\left(\frac{g_{r}}{g_{1}}\right)^{2 / \sqrt{3}} \Omega_{r}(t)\left(\frac{\omega}{\omega_{1}}\right)^{4-2 \nu_{s}}, \quad \omega_{s}<\omega<\omega_{1}
$$




$$
\begin{aligned}
& \sim g_{1}^{2}\left(\frac{g_{r}}{g_{1}}\right)^{2 / \sqrt{3}} \Omega_{r}(t)\left(\frac{\omega}{\omega_{1}}\right)^{4}\left(\frac{\omega_{s}}{\omega_{1}}\right)^{-2 \nu_{s}}, \quad \omega_{r}<\omega<\omega_{s} \\
& \sim g_{1}^{2}\left(\frac{g_{r}}{g_{1}}\right)^{2 / \sqrt{3}} \Omega_{r}(t)\left(\frac{\omega}{\omega_{1}}\right)^{3}\left(\frac{\omega_{s}}{\omega_{1}}\right)^{-2 \nu_{s}}\left(\frac{\omega_{r}}{\omega_{1}}\right), \quad \omega<\omega_{s}
\end{aligned}
$$

(neglecting logarithmic corrections, and using the relation $\omega_{1} / \omega_{r}=\left(H_{1} / H_{r}\right)^{2 / 3}$, valid for the background (4.44)).

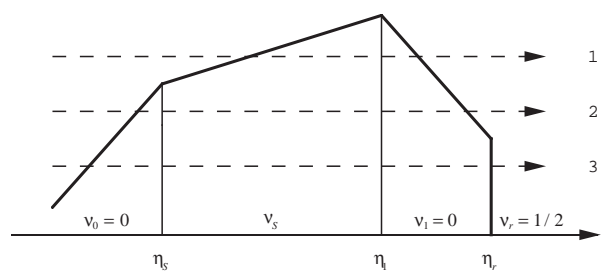

Figure 11: The three branches of the graviton spectrum for the background of Fig. 10, and for the case $\omega_{r}<\omega_{s}$.

For the case $\omega_{s}<\omega_{r}$ the spectrum is similarly obtained from the diagram of Fig. 7. This spectrum differs from that of the minimal model in three important aspects:

- The end point value of the spectrum, $\Omega_{1}\left(\omega_{1}\right)$, is shifted to arbitrarily low values $\sim \Omega_{r} g_{1}^{2(1-1 / \sqrt{3})}<<\Omega_{r}$, since $g_{1}<<g_{r} \sim 1$. The maximal amplified frequency is correspondingly shifted,

$\omega_{1}\left(t_{0}\right)=\frac{\omega_{1}}{\omega_{r}} \frac{H_{r} a_{r}}{a\left(t_{0}\right)} \sim g_{1}^{1 / 2} T_{0}\left(\frac{H_{1}}{H_{r}}\right)^{1 / 6} \sim g_{1}^{1 / 2}\left(\frac{g_{r}}{g_{1}}\right)^{1 / 2 \sqrt{3}} \times 10^{11} \mathrm{~Hz}<<10^{11} \mathrm{~Hz}$

( $T_{0}$ is the present CMB temperature).

- The maximal allowed slope is steeper, $\Omega \sim \omega^{4}$ instead of $\Omega \sim \omega^{3}$.

- The spectrum may be truly non-monotonic, with a peak value at $\omega_{s}$ higher than the end point value, $\Omega\left(\omega_{s}\right)>\Omega\left(\omega_{1}\right)$, provided $4<2 \nu_{s}$.

So, in spite of the fact that the end point is shifted to lower values, the relic background corresponding to a non-minimal model could be detected even more easily than in the minimal case, because of a possible peak at lower frequencies, more accessible to present detectors. It is true that the upper limit, $\Omega_{g} \sim 10^{-2} \Omega_{r}$, cannot be obtained naturally in a non-minimal scenario. However, the analysis of photon production in this scenario shows that such a maximal peak signal would not be excluded by the bounds on the electromagnetic spectrum, provided $\omega_{r}<\omega_{s}$, i.e. provided the post-big bang dilaton-driven phase is long enough with respect to the string phase. It is thus important to point out that, in the context of a 
non-minimal model, the possibility is not excluded to see a graviton background with a peak $\Omega_{G} \sim 10^{-6}$ at an arbitrarily low frequency $\omega_{s}\left(>10^{-8} \mathrm{~Hz}\right.$, however, because of the pulsar bound 48 ), without implying that the spectrum is flat from $\omega_{1}$ to $\omega_{s}$, thus evading the otherwise constraining bound on the total integrated energy following from nucleosynthesis (see eq. (4.14).

\subsection{Experimental sensitivities}

It may be interesting, at this point, to compare the relic graviton background expected in the context of the pre-big bang scenario with the experimental sensitivity of gravity wave detectors. A precise and complete discussion of the present experimental possibilities of detecting a stochastic gravity wave background is, of course, outside the scope of this paper (see for instance the reviews of Refs. $[52,53]$ ). Here I will simply report, at the level of an order of magnitude estimate, the sensitivities accessible to some detectors that are already existing and operating, and some other that are now under construction and will be operating in the near future.

The "theory versus experiments" situation is qualitatively sketched in Fig. 12, where the bold upper border line defines the maximal allowed region for the relic graviton spectrum in a string cosmology context, obtained from Fig. 4; the bold lower line gives the corresponding border in the standard inflationary context.

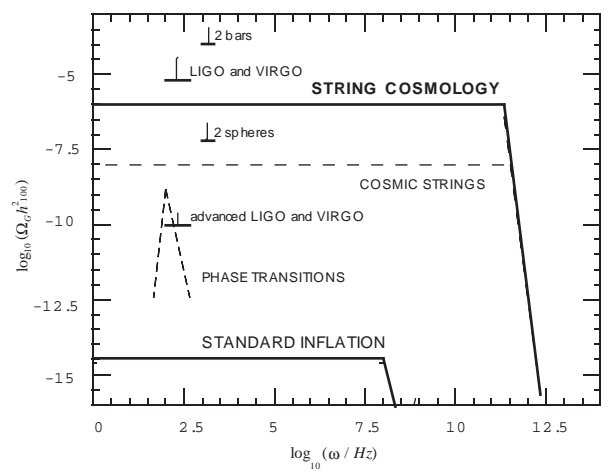

Figure 12: Present and future experimental sensitivities, compared with the allowed region for the graviton spectrum.

Available data, at present, come from the cryogenic, resonant-mass detector EXPLORER, operating at CERN, and provide the upper bound $5 \Omega_{G} \lesssim 500$ at a frequency $\nu=923 \mathrm{~Hz}$, which is too high to be significant for a relic cosmological spectrum. However, by cross-correlating the data obtained from two bar detectors with the same characteristics as those already existing (EXPLORER, NAUTILUS, AURIGA), it will be possible to reach, in very few years, the level of sensitivity 5 $\Omega_{G} \sim 8 \times 10^{-4}$ around the $\mathrm{kHz}$ range. This is still outside, but not so far off, the border of the interesting region, as shown in Fig. 12. Even better sensitivities, in the same frequency range, can be reached through the cross-correlation of a bar and an interferometric detector 5657. And in fact the first generation of interferometric 
detectors, LIGO58 and VIRGO59, when operating, will soon reach a sensitivity 53 $\Omega_{G} \sim 10^{-5}$ centred around the $100 \mathrm{~Hz}$ frequency band. The possible improvement in the sensitivity of microwave cavities quailable at present, operating as gravity wave detectors, is also under active study 60 .

Also shown in Fig. 12 are the sensitivities expected from the cross-correlation of two resonant spherical detectors 55 57, $\Omega_{G} \sim 10^{-7}$ at $\sim 1 \mathrm{kHz}$, and the planned sensitivity of the advanced interferometric detectors361, $\Omega_{G} \sim 10^{-10}$ at $\nu \sim 10^{2} \mathrm{kHz}$. Both are well inside the allowed region, and are thus able to constrain significantly, in case of no detection, the parameters of our class of string cosmology models. To the best of my knowledge, in this range of frequency there are only two other possible primordial backgrounds, competing in intensity with the relics of a pre-big bang phase. The first is the background (with very flat spectrum) generated by cosmic string\$62 or by other topological defects63, characterized by $\Omega_{G} \sim 10^{-8}$. The second is a background sharply peaked around $10^{2}-10^{3} \mathrm{~Hz}$, generated by first order phase transition 62 . 64 in the context of extended inflation models, with peak value $\Omega_{G} \sim 10^{-8}-10^{-9}$. Both these two possibilities are illustrated by the dashed curves of Fig. 12.

Finally, it should be recalled that for non-minimal models of the pre-big bang era, the allowed string cosmology region of Fig. 12 can be extended to the left, in principle without further suppression down to frequencies of $10^{-7} \mathrm{~Hz}$. The space interferometer LISA 65 , operating around the $\mathrm{mHz}$ range, will thus provide significant bounds on non-minimal models and, at the maximal expected sensitivity level of $\Omega_{G} \sim 10^{-10}$, also on minimal models whose spectrum decreases enough to evade the nucleosynthesis bound.

\section{Conclusion}

The cosmological scenario in which I have discussed the production of a relic graviton background was based on two main assumptions:

- the initial state of the Universe is the string perturbative vacuum;

- the pre-big bang phase evolves smoothly into a final decelerated state, dominated by radiation and with constant dilaton.

While the first assumption represents an arbitrary choice of the initial conditions, the second one reflects our present ignorance about string cosmology in the highcurvature and non-perturbative regime, where the transition from the pre- to the post-big bang epoch is expected to occur. Our present lack of knowledge about the kinematics of such a transition forbids, in particular, a precise prediction of the detailed shape of the graviton spectrum.

If we accept the above two assumptions, however, we can already conclude that from a phenomenological point of view the pre-big bang scenario is significantly 
different from the standard inflationary scenario, and that these differences lead to effects that can be tested (at least in principle) even more easily than the corresponding effects of the standard scenario.

In particular, there is no compelling reason in this context to exclude a relic graviton background as high as

$$
\Omega_{g}(\omega) \sim 10^{-6} h_{100}^{-2},
$$

in the whole range from $1 \mathrm{~Hz}$ to $100 \mathrm{GHz}$. Thus, experimental measurements performed at this level of sensitivity would be true tests of Planck-scale physics. The detection of such a cosmic graviton background would represent an event of an importance comparable to the detection of the cosmic black-body radiation 66 . Even more important than this, in some sense, because the CMB photons are relic radiation from the hot, "big bang" phase that occurred in the past history of our Universe. Those gravitons would, instead, be relics of a much earlier "pre-big bang" epoch.

Acknowledgements: This work is supported in part by EC contract ERBCHRXCT94-0488. I am grateful to Ramy Brustein, Massimo Giovannini and Gabriele Veneziano for useful discussions and for fruitful collaboration on the relic graviton background in string cosmology.

\section{Appendix A}

\section{The dimensionless amplitude $\left|\delta_{h}\right|$}

Consider tensor perturbations, in a conformally flat metric background, and in the transverse traceless gauge,

$$
g_{\mu \nu}+\delta g_{\mu \nu}, \quad \delta g_{\mu \nu}=a^{2}(\eta) h_{\mu \nu}, \quad h_{\mu}^{\mu}=0=\nabla_{\nu} h_{\mu}^{\nu} .
$$

The gravitational action, perturbed up to second order in each of the two physical polarization modes $h(x)$,

$$
\frac{M_{p}^{2}}{16 \pi} \int d^{3} x d \eta a^{2}\left[h^{\prime 2}-\left(\nabla_{i} h\right)^{2}\right],
$$

defines the canonical variable 24 26, 28, 60 $u$, which diagonalizes the perturbed action:

$$
u=a h M_{p}
$$

and whose Fourier components $u_{k}$ are required to satisfy canonical commutation relations:

$$
\left[u_{k}, \dot{u}_{k^{\prime}}\right]=i \delta_{k k^{\prime}} .
$$


This fixes the dimensionality of the mode $h_{k},\left[h_{k}\right]=\left[u_{k} / M_{p}\right]=\left[k^{-3 / 2}\right]$, and gives the correct normalization for the Fourier transform in a finite volume $V$,

$$
h(\vec{x}, t)=\frac{1}{\sqrt{V}} \sum_{\vec{k}} h_{\vec{k}}(t) e^{i \vec{k} \cdot \vec{x}} .
$$

In the continuum limit

$$
h(\vec{x}, t)=\frac{\sqrt{V}}{(2 \pi)^{3}} \int d^{3} k h(\vec{k}, t) e^{i \vec{k} \cdot \vec{x}} .
$$

Consider now the two-point correlation function

$$
\xi(\vec{r})=\langle h(\vec{x}) h(\vec{x}+\vec{r})\rangle,
$$

where the brackets denote the quantum expectation value on a given state, if we work in the second-quantization formalism in which the perturbation field is expanded into annihilation and creation operators. We recall, however, that the perturbation background is a stochastic background as a consequence of its quantum origin, since it is obtained by amplifying the quantum fluctuations of the vacuumes, for which

$$
\left\langle a_{k}^{\dagger} a_{k^{\prime}}\right\rangle=|f|^{2} \delta^{3}\left(k-k^{\prime}\right)
$$

where $f$ is an appropriate normalization coefficient. This means that, if we perform the classical limit by replacing quantum expectation values with ensemble averages, we are led to the stochastic condition

$$
\left\langle h(\vec{k}) h\left(-\vec{k}^{\prime}\right)\right\rangle=|h(\vec{k})|^{2} \frac{(2 \pi)^{3}}{V} \delta^{3}\left(k-k^{\prime}\right) .
$$

For a stochastic background the correlation function (A.7) thus becomes

$$
\xi(\vec{r})=\int \frac{d^{3} k}{(2 \pi)^{3}}|h(k)|^{2} e^{-i \vec{k} \cdot \vec{r}}=\frac{1}{2 \pi^{2}} \int \frac{d k}{k} \frac{\sin k r}{k r}\left|\delta_{h}(k)\right|^{2},
$$

where we have defined

$$
\left|\delta_{h}(k)\right|=k^{3 / 2}|h(k)| .
$$

Note that we have used the reality condition, $h^{*}(k)=h(-k)$, and the so-called isotropy condition, i.e. the assumption that $|h(k)|$ is a function only of $k=|\vec{k}|$. The brackets of eq. A.7 can also be formally defined as a macroscopic average over space:

$$
\xi(\vec{r})=\frac{1}{V} \int d^{3} x h(\vec{x}) h(\vec{x}+\vec{r}) ;
$$

the result for $\xi(\vec{r})$ is exactly the same as in eq. (A.10). By evaluating the correlation function for a distance $r=k^{-1}$ we finally obtain from (A.10)

$$
\left[\xi^{1 / 2}(r)\right]_{r=k^{-1}} \sim\left|\delta_{h}(k)\right| .
$$


The variable $\left|\delta_{h}(k)\right|$ can thus be interpreted as the typical, dimensionless amplitude of tensor perturbations over a comoving length scale $r=k^{-1}$.

The spectral density of perturbations, $\Omega_{G}(\omega)$, can be conveniently expressed in terms of $\left|\delta_{h}(k)\right|$ by recalling that the average energy density, summing over polarization, is defined by 34

$$
\rho_{g}=\frac{d E_{g}}{a^{3} d^{3} x}=\frac{M_{p}^{2}}{8 \pi}\left\langle\dot{h}^{2}\right\rangle=-\frac{M_{p}^{2}}{a^{3} 8 \pi} \frac{V}{(2 \pi)^{6}} \int d^{3} k d^{3} k^{\prime} \omega \omega^{\prime} e^{i \vec{x} \cdot\left(\vec{k}+\vec{k}^{\prime}\right)}\left\langle h(k) h\left(k^{\prime}\right)\right\rangle,
$$

where $\omega=|\vec{k}| / a$. By using the stochastic condition (A.9), in the hypothesis $\left|\delta_{h}(k)\right|=\left|\delta_{h}(-k)\right|$,

$$
\frac{d E_{g}}{a^{3} d^{3} x d \ln k}=\frac{M_{p}^{2}}{(2 \pi a)^{3}}\left|\delta_{h}(k)\right|^{2} \omega^{2} .
$$

The spectral distribution of the proper energy density, in critical units of $\rho_{c}=$ $3 M_{p}^{2} H^{2} / 8 \pi$, can thus be expressed as

$$
\frac{d\left(\rho_{g} / \rho_{c}\right)}{d \ln \omega}=\frac{d E_{g}}{\rho_{c} a^{3} d^{3} x d \ln \omega}=\Omega_{G}(\omega, t)=\frac{1}{3 \pi^{2}}\left|\delta_{h}(\omega)\right|^{2}\left(\frac{\omega}{H}\right)^{2} .
$$

\section{Appendix B}

\section{The spectral amplitude $S_{h}$}

Consider the Fourier transform of the polarized gravity wave amplitude $h$,

$$
h(\nu)=\int d t h(t) e^{-2 \pi i \nu t}, \quad \nu=\omega / 2 \pi .
$$

The average proper energy density, summing over polarizations, can be written

$$
\begin{aligned}
\rho_{g} & =\frac{d E_{g}}{a^{3} d^{3} x}=\frac{M_{p}^{2}}{8 \pi}\left\langle\dot{h}^{2}\right\rangle= \\
& =-4 \pi^{2} \frac{M_{p}^{2}}{8 \pi} \int d \nu d \nu^{\prime} \nu \nu^{\prime}\left\langle h(\nu) h\left(\nu^{\prime}\right)\right\rangle e^{2 \pi i\left(\nu+\nu^{\prime}\right) t},
\end{aligned}
$$

where the brackets denote time or ensemble average. By defining the spectral amplitude $S_{h}(\nu)$, such that 69

$$
\left\langle h(\nu) h^{*}\left(-\nu^{\prime}\right)\right\rangle=\frac{1}{2} \delta\left(\nu+\nu^{\prime}\right) S_{h}(\nu),
$$

the energy distribution $\Omega_{g}$, in critical units, can be expressed as

$$
\Omega_{G}(\nu, t)=\frac{1}{\rho_{c}} \frac{d \rho_{G}}{d \ln \nu}=\frac{4 \pi^{2}}{3 H^{2}(t)} \nu^{3} S_{h}(\nu)
$$

(I have assumed $S_{h}(\nu)=S_{h}(-\nu)$ ). 
A particular value of $S_{h}(\nu)$, representing the experimental sensitivity at a given frequency $\nu$, corresponds to a present graviton energy density 43

$$
\Omega_{G}\left(\nu, t_{0}\right) h_{100}^{2} \simeq 1,25 \times 10^{36} \nu^{3} S_{h}(\nu) \mathrm{Hz}^{-2} .
$$

Reaching the level of expected maximal signal, $\Omega_{G} h_{100}^{2} \lesssim 10^{-6}$ (see Sect. 4. 1), would thus require a sensitivity 43

$$
S_{h}^{1 / 2}(\nu) \lesssim 3 \times 10^{-26}\left(\frac{\mathrm{kHz}}{\nu}\right)^{3 / 2} \mathrm{~Hz}^{-1 / 2} .
$$

Comparing eqs. (B.4) and (A.16), we can finally obtain a useful relation between the spectral amplitude $S_{h}$ and the dimensionless amplitude $\delta_{h}$,

$$
S_{h}\left(\frac{\omega}{2 \pi}\right)=\frac{2}{\pi \omega}\left|\delta_{h}(\omega)\right|^{2} .
$$

1. M. Gasperini and G. Veneziano, Astropart. Phys. 1, 317 (1993).

2. M. Gasperini and G. Veneziano, Mod. Phys. Lett. A8, 3701 (1993).

3. M. Gasperini and G. Veneziano, Phys. Rev. D50, 2519 (1994).

4. M. Gasperini, N. Sanchez and G. Veneziano, Nucl. Phys. B364, 365 (1991).

5. N. Sanchez, "String theory in curved spacetime", this book.

6. G. Veneziano, "Classical and quantum string cosmology", this book.

7. E. J. Copeland, A. Lahiri and D. Wands, Phys. Rev. D53, 4247 (1994).

8. I. Antoniadis, J. Rizos and K. Tamvakis, Nucl. Phys. B415, 497 (1994).

9. R. Easther and K. Maeda, "One-loop superstring cosmology and the non-singular Universe", hep-th/9605173.

10. S. J. Rey, "Back reaction and graceful exit in string inflationary cosmology", hep-th/9605176; M. Gasperini and G. Veneziano, "Singularity and exit problems in two-dimensional string cosmology", CERN-TH/96-165, hep-th/9607126.

11. R. Brustein and G. Veneziano, Phys. Lett. B329, 429 (1994); N. Kaloper, R. Madden and K. Olive, Nucl. Phys. B452, 677 (1995); Phys. Lett. B371, 34 (1996); R. Easther, K. Maeda and D. Wands, Phys. Rev. 53, 4247 (1996).

12. M. Gasperini, J. Maharana and G. Veneziano, hep-th/9602087, Nucl. Phys. B (1996), in press; M. Gasperini and G. Veneziano, hep-th/9602096, Gen. Rel Grav. 28 (1996), in press.

13. J. E. Lidsey, "Inflationary and deflationary branches in extended pre-big bang cosmology", gr-qc/9605017.

14. M. Gasperini, M. Giovannini, K. Meissner and G. Veneziano, "Evolution of a string network in background with rolling horizons ", this book (hep-th/9502130).

15. G. Veneziano, Phys. Lett. B265, 287 (1991).

16. A. A. Tseytlin, Mod. Phys. Lett. A6, 1721 (1991).

17. K. A. Meissner and G. Veneziano, Phys. Lett. B267, 33 (1991); Mod. Phys. Lett. A6, 3397 (1991).

18. A. Sen, Phys. Lett. B 271, 295 (1991).

19. M. Gasperini and G. Veneziano, Phys. Lett. B277, 256 (1992). 
20. M. Gasperini, J. Maharana and G. Veneziano, Phys. Lett. B272, 277 (1991); B296, 51 (1992).

21. F. Lucchin and S. Matarrese, Phys. Lett. B164, 282 (1985).

22. L. P. Grishchuk, Sov. Phys. JEPT 40 (1975) 409.

23. A. A. Starobinski, JEPT Lett. 30 (1979) 682.

24. M. Gasperini and M. Giovannini, Phys. Rev. D47, 1519 (1993).

25. R. B. Abbott, B. Bednarz and S. D. Ellis, Phys. Rev. D33, 2147 (1986).

26. R. Brustein, M. Gasperini, M. Giovannini, V. Mukhanov and G. Veneziano, Phys. Rev. D51, 6744 (1995).

27. J. Hwang, Ap. J. 375, 443 (1991).

28. M. Giovannini, PhD Thesis (Turin University, 1996); M. Gasperini and M. Giovannini, "Normal modes for metric fluctuations in a class of anisotropic background", gr-qc/9604002.

29. N. D. Birrel and P. C. W. Davies, "Quantum fields in curved space" (Cambridge Univ. Press, Cambridge, 1982).

30. M. Gasperini, M. Giovannini and G. Veneziano, Phys. Rev. Lett. 75, 3796 (1995).

31. G. F. Smoot et al., Ap. J. 396, L1 (1992); C. L. Bennet et al., Ap. J. 430, 423 (1994).

32. M. Gasperini, M. Giovannini and G. Veneziano, Phys. Rev. D52, 6651 (1995); R. Durrer et al., in preparation.

33. R. Brustein, M. Gasperini, M. Giovannini and G. Veneziano, Phys. Lett. B361, 45 (1995).

34. See for instance E. W. Kolb and M. S. Turner, The Early Universe (Addison-Wesley Publ. Co., New York, 1990).

35. L. M. Krauss and M. White, Phys. Rev. Lett. 69, 869 (1992).

36. D. Polarski and A. A. Starobinski, Class. Quantum Grav. 13, 377 (1996).

37. V. Kaplunovsky, Phys. Rev. Lett. 55, 1036 (1985).

38. L. F. Abbott and M. B. Wise, Nucl. Phys. B244, 54 (1984).

39. B. Allen, Phys. Rev. D37, 2078 (1988).

40. L. P. Grishchuk, Class. Quantum Grav. 10, 2449 (1993).

41. G. Veneziano, private communication.

42. R. Brustein, M. Gasperini and G. Veneziano, in preparation.

43. R. Brustein, M. Gasperini and G. Veneziano, "Peak and end point of the relic graviton background in string cosmology", hep-th/9604804.

44. G. Veneziano, in "String gravity and physics at the Planck energy scale", ed. by N. Sanchez and A. Zichichi (Kluwer Acad. Publ., Dordrecht, 1996), p. 285.

45. M. Gasperini, ibid, p. 305.

46. A. Buonanno, M. Maggiore and C. Ungarelli, "Spectrum of relic gravitational waves in string cosmology", gr-qc/9605072.

47. L. P. Grishchuk, Sov. Phys. Usp. 31, 940 (1988); V. Shani, Phys. Rev. D42, 453 (1990).

48. V. Kaspi, J. Taylor and M. Ryba, Ap. J. 428, 713 (1994).

49. D. H. Lyth, "What would we learn by detecting a gravitational wave signal in the CMB anisotropy?", hep-ph/9606387.

50. M. Abramowitz and I. A. Stegun, "Handbook of mathematical functions" (Dover, New, York, 1972).

51. D. Lemoine and M. Lemoine, Phys. Rev D52, 1955 (1995).

52. M. S. Turner and L. M. Widrow, Phys. Rev. D37, 2743 (1988).

53. B. Allen, "Stochastic gravity-wave background: sources and detectors", grqc/9604033.

54. E. Coccia, "Resonant mass gravitational wave detectors", to appear in Proc. of the 14th Int. Conf. on General Relativity, Florence, August 1995.

55. P. Astone et al., " Upper limit for a gravitational wave stochastic background 
measured with the EXPLORER and NAUTILUS gravitational wave resonant detectors, INFN Preprint, Rome, February 1996, to appear.

56. P. Astone, J. A. Lobo and B. F. Schutz, Class. Quantum Grav. 11, 2093 (1994).

57. S. Vitale, M. Cerdonio, E. Coccia and A. Ortolan, "Gravitational-wave stochastic background detection with resonant-mass detectors", in preparation.

58. A. Abramovici et al., Science 256, 325 (1992).

59. C. Bradaschia et al., Nucl. Instrum. Meth. A289, 518 (1990).

60. E. Picasso, private communication.

61. K. S. Thorne, in " 300 years of gravitation", ed. by S. W. Hawking and W. Israel (Cambridge Univ. Press, Cambridge, 1987), p. 330.

62. R. A. Battye and E. P. S. Shellard, "Primordial gravitational waves: a probe of the very early Universe", astro-ph/9604059.

63. X. Martin and A. Vilenkin, "Gravitational wave background from hybrid topological defects", astro-ph/9606022.

64. M. S. Turner and F. Wilczek, Phys. Rev. Lett. 65, 3080 (1990).

65. J. R. Jafry, J. Cornelisse and R. Reinhard, ESA Journal 8, 219 (1994).

66. E. Penzias and R. W. Wilson, Ap. J. 142, 419 (1965).

67. V. F. Mukhanov, H. A. Feldman and R. H. Brandenberger, Phys. Rep. 215, 203 (1992).

68. L. P. Grishchuk, Phys. Rev. Lett. 70, 2371 (1993).

69. E. Flanagan, Phys. Rev D48, 2389 (1993). 